J. Nonlinear Var. Anal. 2 (2018), No. 2, pp. 131-154

Available online at http://jnva.biemdas.com

https://doi.org/10.23952/jnva.2.2018.2.03

\title{
ON MODULATED ERGODIC THEOREMS
}

\author{
TANJA EISNER ${ }^{1}$, MICHAEL LIN ${ }^{2, *}$ \\ ${ }^{1}$ Institute of Mathematics, University of Leipzig, Germany \\ ${ }^{2}$ Department of Mathematics, Ben-Gurion University, Beer-Sheva, Israel
}

\begin{abstract}
Let $T$ be a weakly almost periodic (WAP) linear operator on a Banach space $X$. A sequence of scalars $\left(a_{n}\right)_{n \geq 1}$ modulates $T$ on $Y \subset X$ if $\frac{1}{n} \sum_{k=1}^{n} a_{k} T^{k} x$ converges in norm for every $x \in Y$. We obtain a sufficient condition for $\left(a_{n}\right)$ to modulate every WAP operator on the space of its flight vectors, a necessary and sufficient condition for (weakly) modulating every WAP operator $T$ on the space of its (weakly) stable vectors, and sufficient conditions for modulating every contraction on a Hilbert space on the space of its weakly stable vectors. We study as an example modulation by the modified von Mangoldt function $\Lambda^{\prime}(n):=\log n 1_{\mathbb{P}}(n)$ (where $\mathbb{P}=\left(p_{k}\right)_{k \geq 1}$ is the sequence of primes), and show that, as in the scalar case, convergence of the corresponding modulated averages is equivalent to convergence of the averages along the primes $\frac{1}{n} \sum_{k=1}^{n} T^{p_{k}} x$. We then prove that for any contraction $T$ on a Hilbert space $H$ and $x \in H$, and also for every invertible $T$ with $\sup _{n \in \mathbb{Z}}\left\|T^{n}\right\|<\infty$ on $L^{r}(\Omega, \mu)$ $(1<r<\infty)$ and $f \in L^{r}$, the averages along the primes converge.

Keywords. Weakly almost periodic operators; Modulated ergodic theorems; Contractions; Hartman sequences; Averaging along the prime numbers; Doubly power-bounded operators on $L^{r}$.
\end{abstract}

2010 Mathematics Subject Classification. 47A35; 47B38; 37A30; 46E30.

\section{INTRODUCTION}

Let $T$ be a power-bounded (linear) operator on a (real or complex) reflexive Banach space $X$. The mean ergodic theorem, proved independently by Lorch, Yosida and Kakutani, says that, for every $x \in X$ the averages $\frac{1}{n} \sum_{k=1}^{n} T^{k} x$ converge in norm.

Definition 1.1. A bounded linear operator $T$ on a Banach space $X$ is said to be (weakly) almost periodic if for every $x \in X$ the orbit $\left\{T^{n} x\right\}_{n \geq 0}$ is (weakly) conditionally compact. A weakly almost periodic operator is necessarily power-bounded. Every power-bounded $T$ on a reflexive Banach space is weakly almost periodic.

The statement of the mean ergodic theorem by Kakutani and by Yosida, and their proofs, show the norm convergence of the averages $\frac{1}{n} \sum_{k=1}^{n} T^{k} x$ for every $x \in X$ when $T$ is weakly almost periodic. The convergence induces a decomposition of the space (the ergodic decomposition)

$$
X=F(T) \oplus \overline{(I-T) X},
$$

where $F(T)$ is the space of fixed points of $T$. The limit operator $E(T)$ is the projection onto $F(T)$ corresponding to the ergodic decomposition.

${ }^{*}$ Corresponding author.

E-mail addresses: tatjana.eisner@math.uni-leipzig.de (T. Eisner), lin@ math.bgu.ac.il (M. Lin).

Received November 23, 2017; Accepted April 10, 2018.

(C) 2018 Journal of Nonlinear and Variational Analysis 
In this article, we are interested in sequences of scalars $\mathbf{a}=\left(a_{k}\right)_{k>0}$ which yield modulated norm ergodic theorems, the strong convergence of the "modulated" averages $\frac{1}{n} \sum_{k=1}^{n} a_{k} T^{k} x$, for every weakly almost periodic $T$ and $x \in X$. Some general results are obtained in Section 2, in particular in Theorem 2.1, which extends a result of [11]. In Section 3, we study modulation of flight vectors and weakly stable vectors of contractions in Hilbert spaces. In Section 4, we treat as an example the modulation by the the modified von Mangoldt function $\Lambda^{\prime}(n)=\log n 1_{\mathbb{P}}(n)$, where $\mathbb{P}=\left\{p_{j}\right\}$ is the set of prime numbers, and prove in particular (Proposition 4.1) that for every power-bounded $T$ on a Banach space, the strong convergence of $\frac{1}{N} \sum_{k=1}^{N} \Lambda^{\prime}(k) T^{k}$ is equivalent to the strong convergence of the averages along the primes $\frac{1}{n} \sum_{j=1}^{n} T^{p_{j}}$. In Theorem 4.3, we prove that for every contraction $T$ on a (real or complex) Hilbert space and $Q(t)$ a polynomial with non-negative integer coefficients, the averages $\frac{1}{n} \sum_{j=1}^{n} T^{Q\left(p_{j}\right)}$ converge strongly. Our main result in Section 5 is the convergence of the averages along the prime powers of doubly power-bounded operators on $L^{r}, 1<r<\infty$ (Theorem 5.3). In Section 6, we list some problems.

\section{Modulated ERGODIC THEOREMS FOR WEAKLY ALMOST PERIODIC OPERATORS}

Definition 2.1. A complex sequence $\mathbf{a}:=\left(a_{k}\right)_{k>0}$ is said be Hartman almost periodic (Hartman for short) if for every $|\lambda|=1$ the limit $c(\lambda):=\lim _{n} \frac{1}{n} \sum_{k=1}^{n} a_{k} \bar{\lambda}^{k}$ exists; $c(\lambda)$ is called the Fourier (or Fourier-Bohr) coefficient function. By Kahane [26], if $\mathbf{a}$ is Hartman, then $\{\lambda: c(\lambda) \neq 0\}$ is countable.

Definition 2.2. Let $T$ be weakly almost periodic on $X$. A vector $x \in X$ is called a fight vector (or weakly almost stable in [15]) if 0 is in the weak closure of its orbit; by the Krein-Shmulian theorem, this is equivalent to $T^{n_{j}} x \stackrel{w}{\rightarrow} 0$ for some subsequence $\left(n_{j}\right)$. The vector $x$ is said to be (weakly) stable (for $T$ ) if $T^{n} x \rightarrow 0$ (weakly). The operator $T$ is said to be (weakly) stable if every $x \in X$ is (weakly) stable for $T$.

A characterization of weakly almost periodic operators on complex Banach spaces is given by the Jacobs-deLeeuw-Glicksberg decomposition [15, Section 16.3], [28, pp. 105-106]:

$$
X=\operatorname{clm}\{y: T y=\lambda y \text { for some } \lambda \in \mathbb{T}\} \oplus\left\{z: T^{n_{j}} z \stackrel{w}{\rightarrow} 0 \text { for some }\left\{n_{j}\right\}\right\} .
$$

Here $\mathrm{clm}$ means the closed linear manifold spanned.

Lemma 2.1. Let $\phi(x): \mathbb{R}^{+} \longrightarrow \mathbb{R}^{+}$be a strictly increasing function with $\lim _{x \rightarrow \infty} \phi(x)=\infty$ and let $\mathbf{a}$ be a complex sequence satisfying

$$
s:=\sup _{n} \frac{1}{n} \sum_{k=1}^{n}\left|a_{k}\right| \phi\left(\left|a_{k}\right|\right)<\infty
$$

Then for every bounded complex sequence $\left(b_{k}\right)_{k>0}$ with $\frac{1}{n} \sum_{k=1}^{n}\left|b_{k}\right| \rightarrow 0$ we have

$$
\frac{1}{n} \sum_{k=1}^{n}\left|a_{k} b_{k}\right| \rightarrow 0 \quad \text { as } n \rightarrow \infty
$$

Proof. When a is bounded the lemma is trivial. Without loss of generality, $\left|b_{k}\right| \leq 1$ for every $k$. For $\varepsilon>0$, taking $C>0$ with $\frac{s}{\phi(C)}<\varepsilon$, and letting $N$ be such that for $n>N$, we have $\frac{1}{n} \sum_{k=1}^{n}\left|b_{k}\right|<\frac{\varepsilon}{C}$. Then, for $n>N$, we have

$$
\frac{1}{n} \sum_{k=1}^{n}\left|a_{k} b_{k}\right|=\frac{1}{n} \sum_{\substack{k=1 \\\left|a_{k}\right| \leq C}}^{n}\left|a_{k} b_{k}\right|+\frac{1}{n} \sum_{\substack{k=1 \\\left|a_{k}\right|>C}}^{n}\left|a_{k}\right| \phi\left(\left|a_{k}\right|\right) \cdot \frac{\left|b_{k}\right|}{\phi\left(\left|a_{k}\right|\right)}<2 \varepsilon .
$$


Remark 2.1. 1. If a satisfies (2.2), then $\sup _{n} \frac{1}{n} \sum_{k=1}^{n}\left|a_{k}\right|<\infty$, since

$$
\frac{1}{n} \sum_{k=1}^{n}\left|a_{k}\right| \leq \frac{1}{n} \sum_{\substack{k=1 \\\left|a_{k}\right| \leq 1}}^{n}\left|a_{k}\right|+\frac{1}{n} \sum_{\substack{k=1 \\\left|a_{k}\right|>1}}^{n}\left|a_{k}\right| \cdot \frac{\phi\left(\left|a_{k}\right|\right)}{\phi(1)} \leq 1+\frac{s}{\phi(1)} .
$$

2. If a satisfies (2.2), then $\left|a_{n}\right|=o(n)$. If not, then for some $c>0$ there exists $\left(n_{j}\right)$ increasing with $\left|a_{n_{j}}\right| \geq c n_{j}$. Then

$$
\frac{1}{n_{j}} \sum_{k=1}^{n_{j}}\left|a_{k}\right| \cdot \phi\left(\left|a_{k}\right|\right) \geq \frac{1}{n_{j}}\left|a_{n_{j}}\right| \cdot \phi\left(\left|a_{n_{j}}\right|\right) \geq c \cdot \phi\left(c n_{j}\right) \rightarrow \infty
$$

contradicting (2.2).

Theorem 2.1. Let $T$ be a weakly almost periodic operator on $X$ and let $\mathbf{a}$ (real if $X$ is over $\mathbb{R}$ ) satisfy (2.2). Then for every flight vector $x$ we have $\lim _{n}\left\|\frac{1}{n} \sum_{k=1}^{n} a_{k} T^{k} x\right\|=0$.

Proof. By Jones and Lin [24], $x$ is a flight vector for $T$ if and only if

$$
\sup _{\left\|x^{*}\right\| \leq 1} \frac{1}{n} \sum_{k=1}^{n}\left|\left\langle x^{*}, T^{k} x\right\rangle\right| \underset{n \rightarrow \infty}{\longrightarrow} 0 .
$$

We may assume $\|x\|=1$. For $x^{*} \in X^{*}$, we put $b_{k}\left(x^{*}\right)=\left|\left\langle x^{*}, T^{k} x\right\rangle\right|$. Then

$$
\left\|\frac{1}{n} \sum_{k=1}^{n} a_{k} T^{k} x\right\|=\sup _{\left\|x^{*}\right\| \leq 1}\left|\frac{1}{n} \sum_{k=1}^{n} a_{k}\left\langle x^{*}, T^{k} x\right\rangle\right| \leq \sup _{\left\|x^{*}\right\| \leq 1} \frac{1}{n} \sum_{k=1}^{n}\left|a_{k} b_{k}\left(x^{*}\right)\right| .
$$

Since (2.6) yields that, for $n>N \sup _{\left\|x^{*}\right\| \leq 1} \frac{1}{n} \sum_{k=1}^{n}\left|b_{k}\left(x^{*}\right)\right|<\frac{\varepsilon}{C}$, the proof of Lemma 2.1 yields that $\sup _{\left\|x^{*}\right\| \leq 1} \frac{1}{n} \sum_{k=1}^{n}\left|a_{k} b_{k}\left(x^{*}\right)\right| \rightarrow 0$. This proves the theorem.

Remark 2.2. The special case of the theorem, with $\phi(x)=x^{p-1}$ for some $p>1$, was proved differently (using Hölder's inequality) by Çömez, Lin and Olsen [11, Theorem 4.1].

Corollary 2.1. Let a be a Hartman sequence satisfying (2.2). Then for every weakly almost periodic operator $T$ on a complex Banach space $X$ and every $x \in X$ the sequence $\frac{1}{n} \sum_{k=1}^{n} a_{k} T^{k} x$ converges in norm.

Proof. We use the decomposition (2.1). Theorem 2.1 applies to the space of flight vectors. For the eigenvectors we have the convergence since $\mathbf{a}$ is Hartman, and on the closed space they generate convergence holds since $\sup _{n}\left\|\frac{1}{n} \sum_{k=1}^{n} a_{k} T^{k}\right\| \leq \sup _{n} \frac{1}{n} \sum_{k=1}^{n}\left|a_{k}\right|$, which is finite by the above remark.

Remark 2.3. It was proved by Lin, Olsen and Tempelman [30, Proposition 1.4] that for every almost periodic $T$ on a complex Banach space $X$ the sequence $\frac{1}{n} \sum_{k=1}^{n} a_{k} T^{k} x$ converges in norm if and only if $\mathbf{a}$ is a Hartman sequence with $\sup _{n} \frac{1}{n} \sum_{k=1}^{n}\left|a_{k}\right|<\infty$. It was shown by Berend, Lin, Rosenblatt and Tempelman [6, Example 2.7] that these necessary conditions on a are not sufficient for the convergence if $T$ is only weakly almost periodic.

Definition 2.3. Let $W_{1}$ be the set of sequences a such that $\|\mathbf{a}\|_{W_{1}}:=\lim \sup _{n} \frac{1}{n} \sum_{k=1}^{n}\left|a_{k}\right|$ is finite. Then $\|\cdot\|_{W_{1}}$ is a semi-norm. We denote by $\mathscr{A}$ the set of sequences a which satisfy (2.2) for some $\phi$ (which by (2.5) are in $W_{1}$ ), and by $\overline{\mathscr{A}}^{W_{1}}$ its closure in the $W_{1}$-semi-norm. By Theorem 2.1 and [30, Theorem 1.2], if $\mathbf{a} \in \overline{\mathscr{A}}^{W_{1}}$, i.e. there are sequences $\mathbf{a}^{(j)}$ which converge in the $W_{1}$-semi-norm to a and each $\mathbf{a}^{(j)}$ satisfies (2.2) with some $\phi_{j}$, then a modulates the flight vectors of every weakly almost periodic operator. 
Lemma 2.2. Let $\left(a_{k}\right)_{k \geq 1}$ be a complex sequence. Then the following are equivalent:

(i) $\sup _{n} \frac{1}{n} \sum_{k=1}^{n}\left|a_{k}\right|<\infty$.

(ii) For every sequence $b_{n} \rightarrow 0$ we have $\frac{1}{n} \sum_{k=1}^{n}\left|a_{k} b_{k}\right| \rightarrow 0$.

(iii) For every sequence $b_{n} \rightarrow 0$ the sequence $\left(\frac{1}{n} \sum_{k=1}^{n} a_{k} b_{k}\right)_{n}$ is bounded.

(iv) For every bounded sequence $\left(b_{n}\right)_{n}$ the sequence $\left(\frac{1}{n} \sum_{k=1}^{n} a_{k} b_{k}\right)_{n}$ is bounded.

Proof. $(i) \Longrightarrow(i i)$ : Let $b_{n} \rightarrow 0$. For $\varepsilon>0$, we take $N$ such that $\left|b_{k}\right|<\varepsilon$ for $k>N$. For $n>N$, we have

$$
\frac{1}{n} \sum_{k=1}^{n}\left|a_{k} b_{k}\right| \leq \frac{1}{n} \sum_{k=1}^{N}\left|a_{k} b_{k}\right|+\varepsilon \frac{1}{n} \sum_{k=N+1}^{n}\left|a_{k}\right|,
$$

which yields (ii).

Trivially, $(i i) \Longrightarrow($ iii) and $(i) \Longrightarrow($ iv $) \Longrightarrow(i i i)$.

$($ iii $) \Longrightarrow(i)$ : Assume (i) fails, so there exists $\left(n_{j}\right)_{j}$ increasing with $\frac{1}{n_{j}} \sum_{k=1}^{n_{j}}\left|a_{k}\right|>j$ for every $j$. Define $\left(b_{n}\right)$ for $n_{j} \leq n<n_{j+1}$ by $\left|b_{n}\right|=1 / \sqrt{j}$ and $\arg b_{n}=-\arg a_{n}$. Then $\left|b_{n}\right| \searrow 0$, and

$$
\left|\frac{1}{n_{j}} \sum_{k=1}^{n_{j}} a_{k} b_{k}\right|=\frac{1}{n_{j}} \sum_{k=1}^{n_{j}}\left|a_{k} b_{k}\right| \geq \frac{1}{\sqrt{j}} \cdot \frac{1}{n_{j}} \sum_{k=1}^{n_{j}}\left|a_{k}\right|>\sqrt{j} \quad \forall j,
$$

contradicting (iii).

Proposition 2.1. Let $\mathbf{a}=\left(a_{k}\right)_{k \geq 1}$ be a sequence of scalars. Then the following are equivalent:

(a) $\sup _{n} \frac{1}{n} \sum_{k=1}^{n}\left|a_{k}\right|<\infty$.

(b) For every bounded linear $T$ on a (real or complex) Banach space $X$ and $x \in X$ weakly stable for $T$ we have $\frac{1}{n} \sum_{k=1}^{n} a_{k} T^{k} x \rightarrow 0$ weakly.

(c) For every bounded linear $T$ on a (real or complex) Banach space $X$ and $x \in X$ stable for $T$ we have $\frac{1}{n} \sum_{k=1}^{n} a_{k} T^{k} x \rightarrow 0$.

Proof. Assume (a). Let $T^{n} x \rightarrow 0$ weakly, and take a functional $x^{*} \in X^{*}$. Putting $b_{n}=\left\langle T^{n} x, x^{*}\right\rangle$ in (ii) of Lemma 2.2, we obtain (b). Similarly, putting $b_{n}=\left\|T^{n} x\right\|$ when $T^{n} x \rightarrow 0$, we obtain (c).

Assume (b). Let $T$ be the shift $T\left(x_{1}, x_{2} \ldots,\right)=\left(x_{2}, x_{3}, \ldots\right)$ on the complex $c_{0}$. Then for every $x \in c_{0}$ we have $T^{n} x \rightarrow 0$. By the assumption, $\frac{1}{n} \sum_{k=1}^{n} a_{k} T^{k} x \rightarrow 0$ weakly for every $x \in c_{0}$, so by the BanachSteinhaus theorem (twice) $\sup _{n}\left\|\frac{1}{n} \sum_{k=1}^{n} a_{k} T^{k}\right\|<\infty$. Now the proof of [30, Proposition 1.3] yields that $\sup _{n} \frac{1}{n} \sum_{k=1}^{n}\left|a_{k}\right|<\infty$.

The proof that (c) implies (a) is similar to (b) implies (a).

Remark 2.4. 1. In (b) we need not have strong convergence; see the example preceding Proposition 3.2.

2. When $T$ is power-bounded, the sets of its weakly stable vectors and of its stable vectors are closed invariant subspaces.

Proposition 2.2. Let $\mathbf{a}$ be a sequence such that for every weakly stable $T$ on a Banach space $X$ and $x \in X$ the sequence $\frac{1}{n} \sum_{k=1}^{n} a_{k} T^{k} x$ converges. Then $\sup _{n} \frac{1}{n} \sum_{k=1}^{n}\left|a_{k}\right|<\infty$ and $\frac{1}{n^{2}} \sum_{k=1}^{n}\left|a_{k}\right|^{2} \rightarrow 0$.

Proof. The first condition follows from the proof of (b) implies (a) in Proposition 2.1. The second condition follows from Theorem 3.1 in the next section.

Proposition 2.3. Let a be a sequence such that for every weakly almost periodic $T$ on a Banach space $X$ and every fight vector $x \in X$ the sequence $\frac{1}{n} \sum_{k=1}^{n} a_{k} T^{k} x$ converges weakly. Then $\sup _{n} \frac{1}{n} \sum_{k=1}^{n}\left|a_{k}\right|<\infty$ and $\frac{1}{n^{2}} \sum_{k=1}^{n}\left|a_{k}\right|^{2} \rightarrow 0$. 
Proof. The first condition follows from the proof of (b) implies (a) in Proposition 2.1. The second condition follows from combining Proposition 3.2 and Lemma 3.1, proved in the next section.

\section{Modulation of Flight vectors of COntractions on a Hilbert space}

It was shown in [30, Theorem 2.1] that if a is Hartman with $\sup _{n} \frac{1}{n} \sum_{k=1}^{n}\left|a_{k}\right|<\infty$, then for every contraction $T$ on a complex Hilbert space $H$ and $x \in H$ the sequence $\frac{1}{n} \sum_{k=1}^{n} a_{k} T^{k} x$ converges in norm; (this is a consequence of Corollary 2.3 of [6], which gives necessary and sufficient conditions). Note that (unlike the general result of [30]) almost periodicity is not required. It is therefore a natural question, when $\mathbf{a}$ is not Hartman, whether for contractions on $H$ the assertion of Theorem 2.1 holds when only $\sup _{n} \frac{1}{n} \sum_{k=1}^{n}\left|a_{k}\right|<\infty$; the example below yields a negative answer.

Proposition 3.1. Let a be a sequence of scalars satisfying $\sup _{n} \frac{1}{n} \sum_{k=1}^{n}\left|a_{k}\right|=C<\infty$. If $\left|a_{k}\right|=o(k)$, then for every contraction $T$ on a (real or complex) Hilbert space $H$ and $x \in H$ with $T^{n} x \stackrel{w}{\rightarrow} 0$ we have $\lim _{n}\left\|\frac{1}{n} \sum_{k=1}^{n} a_{k} T^{k} x\right\|=0$.

Proof. We first prove that $\frac{1}{n} \max _{1 \leq k \leq n}\left|a_{k}\right| \rightarrow 0$ as $n \rightarrow \infty$. For $\varepsilon>0$, there is $K$ such that $\frac{1}{k}\left|a_{k}\right|<\varepsilon$ for $k>K$. For $n>K$, we have

$$
\begin{gathered}
\max _{1 \leq k \leq n}\left(\frac{\left|a_{k}\right|}{n}\right)^{2} \leq \sum_{k=1}^{n} \frac{\left|a_{k}\right|^{2}}{n^{2}} \leq \frac{1}{n^{2}} \sum_{k=1}^{K}\left|a_{k}\right|^{2}+\frac{1}{n} \sum_{k=K+1}^{n}\left|a_{k}\right| \frac{\left|a_{k}\right|}{k} \leq \\
\frac{1}{n^{2}} \sum_{k=1}^{K}\left|a_{k}\right|^{2}+\varepsilon \frac{1}{n} \sum_{k=N+1}^{n}\left|a_{k}\right| \leq \frac{1}{n^{2}} \sum_{k=1}^{K}\left|a_{k}\right|^{2}+C \varepsilon .
\end{gathered}
$$

Hence $\lim _{n \rightarrow \infty} \frac{1}{n} \max _{1 \leq k \leq n}\left|a_{k}\right|=0$. Define $\alpha_{n, 0}=0, \alpha_{n, k}=a_{k} / n$ for $1 \leq k \leq n$, and $\alpha_{n, k}=0$ for $k>n$. Then $\lim _{n} \sup _{k}\left|\alpha_{n, k}\right|=0$ by the above, and Krengel's proof of the generalized Blum-Hanson theorem [28, p. 254] (where in (W5) one should read $c:=\sup _{N} \sum_{i}\left|\alpha_{N i}\right|<\infty$ ) yields the result.

Remark 3.1. Without the additional condition $\left|a_{k}\right|=o(k)$, we have weak convergence in Proposition 3.1, by Proposition 2.1; Theorem 3.1 below shows that in that case strong convergence does not hold.

Corollary 3.1. Let a be a sequence of scalars satisfying $\sup _{n} \frac{1}{n} \sum_{k=1}^{n}\left|a_{k}\right|=C<\infty$. If c (1) $=\lim _{n} \frac{1}{n} \sum_{k=1}^{n} a_{k}$ exists, then for every contraction $T$ on a (real or complex) Hilbert space $H$ with $T^{n}$ convergent in the weak operator topology, necessarily to $E(T)$, we have $\lim _{n}\left\|\frac{1}{n} \sum_{k=1}^{n} a_{k} T^{k} x-c(1) E(T) x\right\|=0$ for every $x \in H$.

Proof. Existence of $c(1)$ yields $\frac{a_{n}}{n}=\frac{1}{n} \sum_{k=1}^{n} a_{k}-\frac{n-1}{n} \cdot \frac{1}{n-1} \sum_{k=1}^{n-1} a_{k} \rightarrow 0$.

Lemma 3.1. Let a satisfy $\sup _{n} \frac{1}{n} \sum_{k=1}^{n}\left|a_{k}\right|=C<\infty$. Then $a_{n}=o(n)$ if and only if $\frac{1}{n^{2}} \sum_{k=1}^{n}\left|a_{k}\right|^{2} \rightarrow 0$.

Proof. One direction follows from $\left|a_{n}\right|^{2} / n^{2} \leq \frac{1}{n^{2}} \sum_{k=1}^{n}\left|a_{k}\right|^{2} \rightarrow 0$. The other direction is shown in (3.1).

Theorem 3.1. Let $H$ be a Hilbert space and let a satisfy $\sup _{n} \frac{1}{n} \sum_{k=1}^{n}\left|a_{k}\right|<\infty$. Then the following are equivalent:

(i) For every weakly stable contraction $T$ on $H$ and $x \in H, \quad\left\|\frac{1}{n} \sum_{k=1}^{n} a_{k} T^{k} x\right\| \rightarrow 0$.

(ii) For some weakly stable unitary operator $U$ on $H$ and some $0 \neq x \in H$ we have $\left\|\frac{1}{n} \sum_{k=1}^{n} a_{k} U^{k} x\right\| \rightarrow 0$. 
(iii) $a_{n}=o(n)$.

(iv) $\frac{1}{n^{2}} \sum_{k=1}^{n}\left|a_{k}\right|^{2} \rightarrow 0$.

Proof. Clearly (i) implies (ii).

Assume (ii). We may assume $\|x\|=1$. The convergence $\left\|\frac{1}{n} \sum_{k=1}^{n} a_{k} U^{k} x\right\| \rightarrow 0$ implies that

$$
\frac{\left|a_{n}\right|}{n}=\frac{\left\|a_{n} U^{n} x\right\|}{n} \rightarrow 0 \text {. }
$$

Since we have $\sup _{n} \frac{1}{n} \sum_{k=1}^{n}\left|a_{k}\right|<\infty$, (iii) implies (i) by Proposition 3.1.

(iv) is equivalent to (iii) by Lemma 3.1.

Remark 3.2. 1 . The condition $\sup _{n} \frac{1}{n} \sum_{k=1}^{n}\left|a_{k}\right|<\infty$ is used only for proving (iii) implies (i). Although it is assumed also in Lemma 3.1, we show that (i) implies (iv) without it: Let $U$ be the shift on $\ell^{2}(\mathbb{Z})$, defined by $U\left(\sum_{j=-\infty}^{\infty} b_{j} \vec{e}_{j}\right)=\sum_{j=-\infty}^{\infty} b_{j} \vec{e}_{j+1}$, where $\left(\vec{e}_{j}\right)_{j \in \mathbb{Z}}$ is the standard orthonormal basis. Then $U$ is a weakly stable unitary operator. By orthogonality of the orbit $\left(U^{k} \vec{e}_{1}\right)$, (i) yields

$$
\frac{1}{n^{2}} \sum_{k=1}^{n}\left|a_{k}\right|^{2}=\left\|\frac{1}{n} \sum_{k=1}^{n} a_{k} U^{k} \vec{e}_{1}\right\|^{2} \rightarrow 0
$$

So (iv) holds.

2. Without the condition $\sup _{n} \frac{1}{n} \sum_{k=1}^{n}\left|a_{k}\right|<\infty$, (iii) does not imply (iv), as shown by the simple example $a_{k}=\sqrt{k}$; since (i) implies (iv), this example shows that without this boundedness condition, (iii) does not imply (i), (and then Proposition 3.1 fails).

3. The condition $\sup _{n} \frac{1}{n} \sum_{k=1}^{n}\left|a_{k}\right|<\infty$ is not necessary for (i); see [6, Example 2.5].

4. The proof of (ii) implies (iii) shows that if we have modulation of one flight vector of an isometry in a Banach space, then $a_{n}=o(n)$. However, we'll show below that this condition, together with the condition $\sup _{n} \frac{1}{n} \sum_{k=1}^{n}\left|a_{k}\right|<\infty$, do not imply modulation of all flight vectors of unitary operators in a (complex) Hilbert space.

5. Condition (iv) is independent of the boundedness of the averages $\frac{1}{n} \sum_{k=1}^{n}\left|a_{k}\right|$. The simple example $a_{k}=k^{1 / 4}$ satisfies (iv) but the averages are unbounded. In the next example the averages are bounded but (iv) fails.

Example 3.1. A sequence a with $\sup _{n} \frac{1}{n} \sum_{k=1}^{n}\left|a_{k}\right|<\infty$ which does not modulate any weakly stable unitary operator on $H$.

We define a non-negative sequence a by $a_{2^{j}}=2^{j-1}$ for $j \geq 1$ and $a_{k}=0$ for all other indices $k \geq 1$. Then for $2^{\ell} \leq n<2^{\ell+1}$ we have

$$
\frac{1}{n} \sum_{k=1}^{n} a_{k} \leq \frac{1}{2^{\ell}} \sum_{j=1}^{\ell} 2^{j-1}=\frac{2^{\ell}-1}{2^{\ell}}<1 .
$$

However, $a_{2^{j}} / 2^{j}=\frac{1}{2}$, so (iii) of the above theorem fails, hence also (ii) fails, so a does not modulate any weakly stable unitary operator.

On the other hand, by Proposition 2.1, $\frac{1}{n} \sum_{k=1}^{n} a_{k} T^{k} x \rightarrow 0$ weakly for every weakly stable operator $T$ on $H$.

Proposition 3.2. If for every unitary operator $T$ on a complex Hilbert space $H$ and every flight vector $x$ we have that $\frac{1}{n} \sum_{k=1}^{n} a_{k} T^{k} x$ converges weakly, then $a_{n}=o(n)$. 
Proof. The assumption implies that for every unitary $T$ and every flight vector $x \in H$, the sequence $\left(\frac{1}{n} \sum_{k=1}^{n} a_{k}\left\langle T^{k} x, x\right\rangle\right)_{n}$ converges, so

$$
\frac{1}{n} a_{n}\left\langle T^{n} x, x\right\rangle \rightarrow 0
$$

Assume that for some $c>0$ and an increasing sequence $\left(n_{j}\right)_{j \geq 1}$ we have $\left|a_{n_{j}}\right| \geq c \cdot n_{j}$, and by taking a subsequence we may assume that $n_{j+1} / n_{j}$ tends to $\infty$. Such a sequence is a rigidity sequence [16, Example 3.4], i.e., there exists a continuous probability $\mu$ on the unit circle $\mathbb{T}$ such that

$$
\lim _{j \rightarrow \infty} \hat{\mu}\left(n_{j}\right)=1 .
$$

For properties and examples of rigidity sequences; see Eisner and Grivaux [16] and Bergelson, Del Junco, Lemanczyk and Rosenblatt [7]. Note that every rigidity sequence has density zero [7, Proposition 2.12].

Let $H:=L^{2}(\mathbb{T}, \mu)$ and define on $H$ the multiplication operator $T$ by $T f(z)=z f(z)$, which is clearly unitary and has no (unimodular) eigenvalues. Hence the function $\mathbf{1}$ is a flight vector for $T$, and $\left\langle T^{n_{j}} \mathbf{1}, \mathbf{1}\right\rangle=$ $\hat{\mu}\left(n_{j}\right) \rightarrow 1$. Hence

$$
\frac{\left|a_{n_{j}}\right|}{n_{j}}\left|\left\langle T^{n_{j}} \mathbf{1}, \mathbf{1}\right\rangle\right| \geq c\left|\left\langle T^{n_{j}} \mathbf{1}, \mathbf{1}\right\rangle\right| \rightarrow c>0
$$

contradicting (3.2).

Remark 3.3. 1. The previous example shows that the proposition is false if we assume the weak convergence only for weakly stable vectors, and not for all flight vectors.

2. Comparing Proposition 3.2 with (ii) implies (iii) in Theorem 3.1, we require in the theorem modulation of one weakly stable vector of a unitary operator, while the proposition requires only weak modulation of flight vectors, but of all unitary operators. On the other hand, the theorem requires $\sup _{n} \frac{1}{n} \sum_{k=1}^{n}\left|a_{k}\right|<\infty$, which is not necssary for (i), and not assumed in the proposition. A sequence without this condition, which satisfies the assumption of the proposition, is given in [6, Example 2.5].

Example 3.2. Sequences a with $\sup \frac{1}{N} \sum_{k=1}^{N}\left|a_{k}\right|<\infty$ which do not modulate flight vectors of a unitary operator.

We produce a way of constructing sequences $\left(a_{n}\right) \subset \mathbb{R}$ with

$$
\sup _{N} \frac{1}{N} \sum_{k=1}^{N}\left|a_{k}\right|<\infty
$$

which fail to be good modulating sequences for unitary operators without eigenvalues.

Let $\left(k_{n}\right) \subset \mathbb{N}$ be a rigidity sequence. Define $k_{0}:=0$ and

$$
\left|a_{n}\right|:= \begin{cases}k_{l}-k_{l-1}, & n=k_{l} \text { for } l \geq 1, \\ 0, & \text { otherwise. }\end{cases}
$$

Then for $k_{n} \leq N<k_{n+1}$ we have

$$
\frac{1}{N} \sum_{l=1}^{N}\left|a_{l}\right| \leq \frac{1}{k_{n}} \sum_{l=1}^{k_{n}}\left|a_{l}\right|=\frac{1}{k_{n}} \sum_{l=1}^{n}\left|a_{k_{l}}\right|=\frac{1}{k_{n}} \sum_{l=1}^{n}\left(k_{l}-k_{l-1}\right)=1
$$

and (3.4) is satisfied. 
Consider now the complex Hilbert space $L^{2}(\mathbb{T}, \mu)$ with the multiplicaton operator $T$ defined on it by $(T f)(z):=z f(z)$. Since $\hat{\mu}(n)=\left\langle T^{n} \mathbf{1}, \mathbf{1}\right\rangle, n \in \mathbb{N}$, it is enough to make the averages

$$
\frac{1}{N} \sum_{k=1}^{N} a_{k} \hat{\mu}(k)
$$

diverge. We will define a rule for each $s_{n}:=\operatorname{sign}\left(a_{k_{n}}\right)$ to be \pm 1 later. Observe

$$
\frac{1}{k_{n}} \sum_{l=1}^{k_{n}} a_{l} \hat{\mu}(l)=\frac{1}{k_{n}} \sum_{l=1}^{n} a_{k_{l}} \hat{\mu}\left(k_{l}\right) .
$$

By (3.3) and (3.5), Lemma 2.2(ii) yields that the convergence of the right hand side of (3.6) is equivalent to the convergence of

$$
\frac{1}{k_{n}} \sum_{l=1}^{n} a_{k_{l}}=\frac{1}{k_{n}} \sum_{l=1}^{n}\left(k_{l}-k_{l-1}\right) s_{l},
$$

which is the $k_{n}$ th Cesàro average of the sequence

$$
s_{1}, \ldots, s_{1}, s_{2}, \ldots, s_{2}, s_{3}, \ldots, s_{3}, \ldots
$$

where $s_{l}$ appears exactly $k_{l}-k_{l-1}$ times. Now it is easy to define $s_{l}$ to be 1 or -1 such that these averages diverge (first make the averages close to 1 , then close to -1 etc.). Note that every such sequence $\left(a_{n}\right)$ is by construction Cesàro divergent and therefore not Hartman. As a concrete example, one can take $k_{n}:=2^{n}$, which is a rigidity sequence by Eisner and Grivaux [16, Prop. 3.9], or Bergelson, Del Junco, Lemanczyk and Rosenblatt [7, Prop. 3.27].

Example 3.3. A sequence a with $\sup \frac{1}{N} \sum_{k=1}^{N}\left|a_{k}\right|<\infty$ and $a_{k}=o(k)$ which does not modulate flight vectors of a unitary operator.

In the previous example, take a rigidity sequence $\left(k_{n}\right)$ with $\lim _{n \rightarrow \infty} \frac{k_{n+1}}{k_{n}}=1$, which exists by [16, Example 3.17]. Then, in addition to (3.4), $\left(a_{n}\right)$ satisfies $a_{n}=o(n)$, since

$$
\frac{\left|a_{k_{n}}\right|}{k_{n}}=\frac{k_{n}-k_{n-1}}{k_{n}}=1-\frac{k_{n-1}}{k_{n}} \rightarrow 0 \quad \text { as } n \rightarrow \infty .
$$

This example shows that good modulating sequences for weakly stable operators (as in Proposition 3.1) need not modulate flight vectors of all unitary operators. In particular, the sequence in this example does not satisfy (2.2) for any $\phi$ as in Lemma 2.1.

Remark 3.4. Note that $\left(k_{n}\right)$ is a rigidity sequence if and only if there exists a weakly mixing system $(\Omega, v, T)$ which is rigid along $\left(k_{n}\right)$, i.e., $\lim _{n \rightarrow \infty} T^{k_{n}}=I$ in the strong operator topology. (In fact, this is one of the several equivalent definitions of rigidity sequences). So the unitary operator can be constructed to be the Koopman operator of a weakly mixing transformation restricted to the orthogonal complement of the function 1 and the averages

$$
\frac{1}{N} \sum_{k=1}^{N} a_{k} T^{k} f
$$

diverge for every non-zero $f$. 


\section{EXAmple: Modulation By the VOn MangOldT FUnCTION}

Weighted ergodic theorems for dynamical systems, where the weights are given by some arithmetic function, were obtained by Cuny and Weber [13]. In weighted ergodic theorems, we have a non-negative sequence of weights $\left(w_{n}\right)$ with diverging partial sums $W_{n}:=\sum_{k=1}^{n} w_{k}$, and one considers convergence of the weighted averages $\frac{1}{W_{n}} \sum_{k=1}^{n} w_{k} T^{k} f$. When $W_{n} / n$ converges to a (finite) non-zero limit, convergence of weighted averages and of averages modulated by $\left(w_{n}\right)$ coincide.

In this section, we consider modulation by the von Mangoldt function $\Lambda$, defined by

$$
\Lambda(n):= \begin{cases}\log p, & n=p^{k} \text { for some } p \in \mathbb{P}, k \in \mathbb{N}, \\ 0, & \text { otherwise }\end{cases}
$$

and by its simplified version $\Lambda^{\prime}$ defined by $\Lambda^{\prime}(n):=1_{\mathbb{P}}(n) \log n$ (P्P denotes the set of primes). The prime number theorem implies $\lim _{N \rightarrow \infty} \frac{1}{N} \sum_{k=1}^{N} \Lambda(k)=1$ [14, pp. 56,118] [3, p. 79], and also

$$
\lim _{N \rightarrow \infty} \frac{1}{N} \sum_{k=1}^{N} \Lambda^{\prime}(k)=1
$$

Hence (see also [19, formula (5)]),

$$
\lim _{N \rightarrow \infty}\left|\frac{1}{N} \sum_{k=1}^{N} \Lambda(k)-\frac{1}{N} \sum_{k=1}^{N} \Lambda^{\prime}(k)\right|=\lim _{N \rightarrow \infty} \frac{1}{N} \sum_{k=1}^{N}\left(\Lambda(k)-\Lambda^{\prime}(k)\right)=0 .
$$

The proof of the next lemma is included for the sake of completeness (making precise the proof of [19, Lemma 1]).

Lemma 4.1. For every $C>0$, we have

$$
\lim _{N \rightarrow \infty} \sup _{\left\|\left(b_{k}\right)\right\|_{\infty} \leq C}\left|\frac{1}{N} \sum_{k=1}^{N} \Lambda^{\prime}(k) b_{k}-\frac{1}{\pi(N)} \sum_{p \leq N, p \in \mathbb{P}} b_{p}\right|=0,
$$

where $\pi(N)$ is the number of primes not exceeding $N$.

Proof. Let $p_{1}, p_{2}, \ldots$ be the sequence of primes in increasing order. By the prime number theorem, $\lim _{N} \frac{\pi(N) \log N}{N}=1$, so for $\varepsilon>0$ there exists $N_{0}$ such that $\frac{\pi(N) \log N}{N}<1+\varepsilon$ for $N>N_{0}$. For $N>N_{1} \geq N_{0}$ also $\left|1-\frac{1}{N} \sum_{j=1}^{\pi(N)} \log p_{j}\right|<\varepsilon$, by (4.1). For $\left(b_{k}\right)$ bounded with $\left\|\left(b_{k}\right)\right\|_{\infty} \leq C$ and $N>N_{1}$ we therefore have

$$
\begin{gathered}
\left|\frac{1}{\pi(N)} \sum_{p \leq N, p \in \mathbb{P}} b_{p}-\frac{1}{N} \sum_{k=1}^{N} \Lambda^{\prime}(k) b_{k}\right|=\left|\sum_{j=1}^{\pi(N)}\left[\frac{1}{\pi(N)}-\frac{\log p_{j}}{N}\right] b_{p_{j}}\right| \leq \\
\left|\sum_{j=1}^{\pi(N)}\left[\frac{1+\varepsilon}{\pi(N)}-\frac{\log p_{j}}{N}\right] b_{p_{j}}\right|+\varepsilon\left|\frac{1}{\pi(N)} \sum_{j=1}^{\pi(N)} b_{p_{j}}\right| \leq \\
\sum_{j=1}^{\pi(N)}\left[\frac{1+\varepsilon}{\pi(N)}-\frac{\log p_{j}}{N}\right]\left\|\left(b_{k}\right)\right\|_{\infty}+\varepsilon\left\|\left(b_{k}\right)\right\|_{\infty}= \\
\left(1-\frac{1}{N} \sum_{j=1}^{\pi(N)} \log p_{j}\right)\left\|\left(b_{k}\right)\right\|_{\infty}+2 \varepsilon\left\|\left(b_{k}\right)\right\|_{\infty} \leq 3 \varepsilon\left\|\left(b_{k}\right)\right\|_{\infty} \leq 3 \varepsilon C .
\end{gathered}
$$

This shows that

$$
\sup _{\left\|\left(b_{k}\right)\right\|_{\infty} \leq C}\left|\frac{1}{\pi(N)} \sum_{p \leq N, p \in \mathbb{P}} b_{p}-\frac{1}{N} \sum_{k=1}^{N} \Lambda^{\prime}(k) b_{k}\right| \underset{N \rightarrow \infty}{\longrightarrow} 0 .
$$


Remark 4.1. By (4.2) and (4.3), for every $C>0$, we have also

$$
\lim _{N \rightarrow \infty} \sup _{\left\|\left(b_{k}\right)\right\|_{\infty} \leq C}\left|\frac{1}{N} \sum_{k=1}^{N} \Lambda(k) b_{k}-\frac{1}{\pi(N)} \sum_{p \leq N, p \in \mathbb{P}} b_{p}\right|=0 .
$$

Proposition 4.1. The following are equivalent for a power-bounded operator $T$ on a Banach space $X$ and $x \in X$ :

(i) $\frac{1}{n} \sum_{k=1}^{n} T^{p_{k}} x$ converges.

(ii) $\frac{1}{N} \sum_{j=1}^{\pi(N)} \log p_{j} T^{p_{j}} x=\frac{1}{N} \sum_{k=1}^{N} \Lambda^{\prime}(k) T^{k} x$ converges.

(iii) $\frac{1}{N} \sum_{k=1}^{N} \Lambda(k) T^{k} x$ converges.

If either limit exists, then the three limits are the same.

Proof. To prove the equivalence of (i) and (ii), let $M:=\sup _{n}\left\|T^{n}\right\|$, and fix $x \in X$. For $x^{*} \in X^{*}$, put $b_{k}\left(x^{*}\right)=\left\langle x^{*}, T^{k} x\right\rangle$. Then (4.4) yields

$$
\begin{gathered}
\left\|\frac{1}{\pi(N)} \sum_{k=1}^{\pi(N)} T^{p_{k}} x-\frac{1}{N} \sum_{k=1}^{N} \Lambda^{\prime}(k) T^{k} x\right\|=\sup _{\left\|x^{*}\right\| \leq 1}\left|\frac{1}{\pi(N)} \sum_{k=1}^{\pi(N)} b_{p_{k}}\left(x^{*}\right)-\frac{1}{N} \sum_{k=1}^{N} \Lambda^{\prime}(k) b_{k}\left(x^{*}\right)\right| \leq \\
\sup _{\left\|\left(b_{k}\right)\right\|_{\infty} \leq M\|x\|}\left|\frac{1}{\pi(N)} \sum_{p \leq N, p \in \mathbb{P}} b_{p}-\frac{1}{N} \sum_{k=1}^{N} \Lambda^{\prime}(k) b_{k}\right| \rightarrow 0 .
\end{gathered}
$$

For the equivalence of (ii) and (iii), we use (4.2). Since $\Lambda(k) \geq \Lambda^{\prime}(k)$, we obtain

$$
\begin{gathered}
\left\|\frac{1}{N} \sum_{k=1}^{N} \Lambda(k) T^{k} x-\frac{1}{N} \sum_{k=1}^{N} \Lambda^{\prime}(k) T^{k} x\right\| \leq \frac{1}{N} \sum_{k=1}^{N}\left\|\left(\Lambda(k)-\Lambda^{\prime}(k)\right) T^{k} x\right\| \leq \\
\left(\frac{1}{N} \sum_{k=1}^{N} \Lambda(k)-\frac{1}{N} \sum_{k=1}^{N} \Lambda^{\prime}(k)\right) M\|x\| \rightarrow 0 .
\end{gathered}
$$

Our proof shows that if either limit exists, then the three limits are the same.

Remark 4.2. 1 . The above proof shows also the equivalence of the above three conditions when the convergence is taken weakly.

2. The equivalence of (i) with (ii), or (iii), transforms the averages along the primes into modulated (or, by (4.1), weighted) averages.

For $\lambda \in \mathbb{T}$, (4.2) yields $\lim _{N}\left|\frac{1}{N} \sum_{k=1}^{N} \Lambda(k) \lambda^{k}-\frac{1}{N} \sum_{k=1}^{N} \Lambda^{\prime}(k) \lambda^{k}\right|=0$, so $(\Lambda(n))$ is Hartman if and only if $\left(\Lambda^{\prime}(n)\right)$ is, and in that case they have the same Fourier-Bohr coefficients.

Theorem 4.1. (i) The sequences $(\Lambda(n))$ and $\left(\Lambda^{\prime}(n)\right)$ are Hartman, and have the same Fourier-Bohr coefficients $c(\lambda)$.

(ii) For every contraction $T$ on a complex Hilbert space $H$ and $x \in H$, we have the convergence

$$
\begin{gathered}
\lim _{n \rightarrow \infty} \frac{1}{n} \sum_{j=1}^{n} T^{p_{j}} x=\lim _{N \rightarrow \infty} \frac{1}{N} \sum_{j=1}^{\pi(N)} \log p_{j} T^{p_{j}} x=\lim _{N \rightarrow \infty} \frac{1}{N} \sum_{k=1}^{N} \Lambda(k) T^{k} x= \\
\sum_{\lambda \in \sigma(\Lambda(n))} c(\lambda) E(\bar{\lambda}) x
\end{gathered}
$$

where $E(\lambda)$ is the orthogonal projection on the eigenspace corresponding to $\lambda \in \mathbb{T}$. 
In particular, for every flight vector $x \in H$,

$$
\lim _{n \rightarrow \infty} \frac{1}{n} \sum_{j=1}^{n} T^{p_{j}} x=\lim _{N \rightarrow \infty} \frac{1}{N} \sum_{j=1}^{\pi(N)} \log p_{j} T^{p_{j}} x=\lim _{N \rightarrow \infty} \frac{1}{N} \sum_{k=1}^{N} \Lambda(k) T^{k} x=0 .
$$

Proof. We first prove the assertion (i) for $\left(\Lambda^{\prime}(n)\right)$. By (4.1), $\lim _{N \rightarrow \infty} \frac{1}{N} \sum_{k=1}^{N} \Lambda^{\prime}(k)=1$, so by positivity, $\sup _{N} \frac{1}{N} \sum_{k=1}^{N}\left|\Lambda^{\prime}(k)\right|<\infty$.

It is easily checked that $\frac{1}{\pi(N)} \sum_{p \leq N, p \in \mathbb{P}} \lambda^{p}=\frac{1}{\pi(N)} \sum_{k=1}^{\pi(N)} \lambda^{p_{k}}$ for $\lambda \in \mathbb{T}$. The uniform distribution mod 1 of $\left(p_{k} \theta\right)$ for every $\theta$ irrational, proved by Vinogradov, is equivalent (by Weyl's criterion) to the convergence to zero of $\frac{1}{n} \sum_{k=1}^{n} \lambda^{p_{k}}$ for every $\lambda \in \mathbb{T}$ not a root of unity, which yields $\frac{1}{\pi(N)} \sum_{p \leq N, p \in \mathbb{P}} \lambda^{p} \rightarrow 0$ for such $\lambda$; see also [12, Lemma 4.1]. For $\lambda \in \mathbb{T}$ a root of unity, dividing by $\pi(N)$ formula (3.5) in [37, p. 180], we obtain that $\frac{1}{\pi(N)} \sum_{p \leq N, p \in \mathbb{P}} \lambda^{p}$ converges (with an identification of the limit).

Hence, by (4.3), for every $\lambda \in \mathbb{T}$ there exists the limit

$$
c(\lambda):=\lim _{N \rightarrow \infty} \frac{1}{N} \sum_{k=1}^{N} \Lambda^{\prime}(k) \lambda^{k}=\lim _{N \rightarrow \infty} \frac{1}{\pi(N)} \sum_{p \leq N, p \in \mathbb{P}} \lambda^{p} .
$$

This shows that $\left(\Lambda^{\prime}(n)\right)$ is Hartman. (Note that $c(\lambda)=0$ for every $\lambda$ which is not a root of unity; for an explicit formula when $\lambda$ is a root of unity see, e.g., [37, p. 180] or [12, equation (7)]). Since $\Lambda(n) \geq \Lambda^{\prime}(n)$, we conclude by (4.2) that also $(\Lambda(n))$ is Hartman, with the same coefficients $c(\lambda)$.

(ii) The convergence of $\frac{1}{n} \sum_{k=1}^{n} \lambda^{p_{k}}$ for every $\lambda \in \mathbb{T}$ obtained above yields, by [30, Theorem 4.4], that for every contraction $T$ on a Hilbert space $\frac{1}{n} \sum_{k=1}^{n} T^{p_{k}} x$ converges strongly for every $x \in H$, and since in (4.6) $c(\lambda) \neq 0$ only for countably many $\lambda$ (roots of unity),

$$
\lim _{N \rightarrow \infty} \frac{1}{\pi(N)} \sum_{j=1}^{\pi(N)} T^{p_{j}} x=\lim _{N \rightarrow \infty} \frac{1}{N} \sum_{k=1}^{N} T^{p_{k}} x=\sum_{\lambda \in \mathbb{T} \text { root of } 1} c(\lambda) E(\bar{\lambda}) x, \quad \forall x \in H .
$$

Existence of the other limits in (ii) and their equality follow from Proposition 4.1.

Remark 4.3. 1 . If $T$ is invertible on $H$ with $T$ and $T^{-1}$ both power-bounded, then the assertion of (ii) holds, since by Sz.-Nagy [40] $T$ is similar to a unitary operator.

2. If $T$ is almost periodic on a complex Banach space, the averages along the primes $\frac{1}{\pi(N)} \sum_{j=1}^{\pi(N)} T^{p_{j}} x$ converge strongly, because for $T$ almost periodic $x \in X$ is a flight vector if and only if $\left\|T^{n} x\right\| \rightarrow 0$, and convergence for the eigenvectors follows from (4.6). Proposition 4.1 yields convergence of the modulated averages $\frac{1}{N} \sum_{k=1}^{N} \Lambda(k) T^{k} x$ and $\frac{1}{N} \sum_{k=1}^{N} \Lambda^{\prime}(k) T^{k} x$.

Theorem 4.2. Let $T$ be a contraction on a real Hilbert space $H$. Then for every $x \in H$ we have norm convergence of $\frac{1}{n} \sum_{j=1}^{n} T^{p_{j}} x$ and of $\frac{1}{N} \sum_{j=1}^{\pi(N)} \log p_{j} T^{p_{j}} x$.

Proof. Given a real Hilbert space $H$, we define its complexification $H_{C}:=H \times H$ with the usual addition of pairs, and multiplication by complex scalars defined by

$$
(\alpha+i \beta)(x, y):=(\alpha x-\beta y, \alpha y+\beta x) .
$$

A scalar product on $H_{C}$ is defined by

$$
\langle(x, y),(u, v)\rangle:=\langle x, u\rangle+\langle y, v\rangle+i(\langle y, u\rangle-\langle x, v\rangle),
$$


so the norm on $H_{C}$ is given by $\|(x, y)\|^{2}=\|x\|^{2}+\|y\|^{2}$ (see [32, p. 98] for proofs). Given a linear operator $T$ on (the real) $H$ we define its extension to $H_{C}$ by $T_{C}(x, y):=(T x, T y)$. Clearly $\left\|T_{C}\right\| \leq\|T\|$, and since $\left\|T_{C}(x, 0)\right\|=\|T x\|$, we have $\left\|T_{C}\right\|=\|T\|$.

Given a contraction on the real Hilbert space $H$, we apply Theorem 4.1(ii) to $T_{C}$ on $H_{C}$ and obtain the assertion of our theorem.

Remark 4.4. 1. The deep result of Bourgain [8], [9] and Wierdl [43] on the pointwise ergodic theorem along primes for $f \in L^{r}(r>1)$ of a probability preserving system (together with Wierdl's [43, Lemma 1]) shows that for any probability preserving system $(\Omega, \Sigma, \mu, \tau)$ and $f \in L^{r}(\Omega, \mu), r>1$, we have that $\frac{1}{N} \sum_{j=1}^{\pi(N)} \log p_{j} f\left(\tau^{p_{j}} \omega\right)$ converges a.e. (see formula (2) in [43]), i.e. $\left(\Lambda^{\prime}(n)\right)$ is in fact a good weight sequence for the pointwise ergodic theorem in $L^{r}$. For $r=2$ the limit is identified by the series in (4.5) (where $T f=f \circ \tau$ ). It can be shown that also $(\Lambda(n))$ is a good weight sequence for the pointwise ergodic theorem in $L^{r}$.

2. For pointwise ergodic theorems with other arithmetic weights see El Abdalaoui, Kulaga-Przymus, Lemańczyk, de la Rue [17, Section 3] and Cuny, Weber [13].

3. In his thesis [44], Wierdl extended the result of [43] to a.e. convergence of averages of $L^{2}$ functions along fixed powers of primes. This result was rediscovered by Nair [35] (also for $L^{2}$ functions), and extended in [36] to averages of $L^{r}$ functions $(r>1)$ along $Q\left(p_{j}\right)$, where $Q(t)$ is a polynomial with nonnegative integer coefficients.

Theorem 4.3. Let $Q(t)$ be a polynomial with non-negative integer coefficients. Then for every contraction $T$ on a (real or complex) Hilbert space $H$ and $x \in H$, the averages $\frac{1}{n} \sum_{j=1}^{n} T^{Q\left(p_{j}\right)} x$ converge in norm.

Proof. We first prove the theorem for a complex Hilbert space $H$. For $\lambda=e^{2 \pi i \alpha} \in \mathbb{T}$ which is not a root of unity (i.e $\alpha$ irrational), the sequence $\left(\alpha Q\left(p_{j}\right)\right)$ is equidistributed modulo 1, by Vinogradov (see Rhin [38]), so by Weyl's criterion $\frac{1}{n} \sum_{j=1}^{n} \lambda Q\left(p_{j}\right) \rightarrow 0$.

For a root of unity $\lambda=e^{2 \pi i \frac{b}{q}}$ with $(b, q)=1$, note that if $p \equiv a$ modulo $q$, then $\lambda^{Q(p)}=\lambda^{Q(a)}$. We now use the prime number theorem in arithmetic progressions [42, p. 598] (see also [14, p. 133]). Let $\pi(x, q, a):=|\{p \in \mathbb{P}: p \leq x, p \equiv a \bmod q\}|$ and denote $\operatorname{Li}(x):=\int_{2}^{x} \frac{1}{\log t} d t$. If $(a, q)=1$, then for some $s<1$, for large $x$ we have

$$
\left|\pi(x, q, a)-\frac{\operatorname{Li}(x)}{\phi(q)}\right| \leq A \frac{x}{\mathrm{e}^{A \sqrt{x}}}+A \frac{x^{s}}{\log x} .
$$

Using the notation $e(x):=\mathrm{e}^{2 \pi i x}$, we have

$$
\begin{gathered}
\frac{1}{N} \sum_{j=1}^{N} \lambda^{Q\left(p_{j}\right)=\frac{1}{N}} \sum_{j=1}^{N} e\left(Q\left(p_{j}\right) \frac{b}{q}\right)=\sum_{a=0}^{q-1} \frac{1}{N} \sum_{\substack{j \leq N \\
p_{j} \equiv a} \bmod q} e\left(Q(a) \frac{b}{q}\right)= \\
\sum_{a=0}^{q-1} e\left(Q(a) \frac{b}{q}\right) \cdot \frac{\pi\left(p_{N}, q, a\right)}{N} .
\end{gathered}
$$

Since $\pi(x, q, a)$ is at most 1 when $(a, q)>1$, we compute the limit as $N \rightarrow \infty$ for $(a, q)=1$; since $\frac{\operatorname{Li}(x)}{\pi(x)} \rightarrow 1$ as $x \rightarrow \infty$ (e.g. [3, p. 102]) and $\pi\left(p_{N}\right)=N$, (4.7) yields

$$
\lim _{N \rightarrow \infty} \frac{\pi\left(p_{N}, q, a\right)}{N}=\frac{1}{\phi(q)} \lim _{N \rightarrow \infty} \frac{\operatorname{Li}\left(p_{N}\right)}{\pi\left(p_{N}\right)}=\frac{1}{\phi(q)},
$$


so that

$$
\lim _{N \rightarrow \infty} \frac{1}{N} \sum_{j=1}^{N} \lambda^{Q\left(p_{j}\right)}=\frac{1}{\phi(q)} \sum_{\substack{0<a<q \\(a, q)=1}} e\left(Q(a) \frac{b}{q}\right) .
$$

We therefore have that for every $\lambda \in \mathbb{T}$ the sequence $\frac{1}{n} \sum_{j=1}^{n} \lambda Q\left(p_{j}\right)$ converges. By [30, Theorem 4.4], $\frac{1}{n} \sum_{j=1}^{n} T^{Q\left(p_{j}\right)} x$ converges for every contraction $T$ on $H$ and $x \in H$.

When $H$ is a real Hilbert space, we use the complexification, as in the proof of Theorem 4.2, to deduce the result from the complex case.

Remark 4.5. A quick proof for the complex case, based on later results in ergodic theory, is this: For any $\lambda \in \mathbb{T}$, we apply Nair's result [36, Theorem 1] to the unit circle rotation $\theta(z)=\lambda z$ and the function $f(z)=z$, and deduce that $\frac{1}{n} \sum_{j=1}^{n} \lambda Q\left(p_{j}\right)$ converges. Theorem 4.4 of [30] yields that if $T$ is a contraction on $H$, the desired convergence holds.

By Theorems 4.1 and 4.2, $\left(\Lambda^{\prime}(n)\right)_{n}$ is a Hartman sequence which modulates all contractions in a (real or complex) Hilbert space. The following shows that $\left(\Lambda^{\prime}(n)\right)_{n}$ cannot be approximated in the $W_{1}$-seminorm by sequences satisfying (2.2). Thus the set of sequences which modulate all flight vectors of Hilbert space contractions is strictly larger than $\overline{\mathscr{A}}^{W_{1}}$ defined in Section 2.

Proposition 4.2. Every $\left(a_{n}\right) \in \mathscr{A}$ satisfies $\left\|a_{n}-\Lambda^{\prime}(n)\right\|_{W_{1}} \geq \frac{1}{2}$. In particular, both $\left(\Lambda^{\prime}(n)\right)$ and $(\Lambda(n))$ do not belong to $\overline{\mathscr{A}}^{W_{1}}$.

Proof. Let $\left(a_{n}\right)$ satisfy

$$
d:=\left\|\left(a_{n}\right)-\Lambda^{\prime}\right\|_{W_{1}}<\frac{1}{2} .
$$

We have to show that $\left(a_{n}\right) \notin \mathscr{A}$. Define $\left(b_{n}\right)$ by

$$
b_{n}:=\left|a_{n}\right| 1_{M}(n),
$$

where

$$
M:=\left\{p \in \mathbb{P}:\left|a_{p}\right| \geq \frac{\log p}{2}\right\} .
$$

Observe

$$
\left|b_{n}-\Lambda^{\prime}(n)\right|= \begin{cases}|| a_{n}\left|-\Lambda^{\prime}(n)\right| \leq\left|a_{n}-\Lambda^{\prime}(n)\right|, & n \in M, \\ \log n \leq 2\left|a_{n}-\Lambda^{\prime}(n)\right|, & n \in \mathbb{P} \backslash M, \\ 0, & n \notin \mathbb{P} .\end{cases}
$$

Therefore $\left(b_{n}\right)$ satisfies

$$
\left\|\left(b_{n}\right)-\Lambda^{\prime}\right\|_{W_{1}} \leq 2 d<1 .
$$

Moreover, since $\left(b_{n}\right)$ is dominated by $\left(\left|a_{n}\right|\right)$, it suffices to show that $\left(b_{n}\right) \notin \mathscr{A}$.

Let $\phi: \mathbb{R}_{+} \rightarrow \mathbb{R}_{+}$satisfy $\phi \nearrow \infty$ and fix $C>0$. Observe that $b_{p} \geq C$ for every $p \in M$ with $p \geq e^{2 C}$ and therefore for $N>e^{2 C}$ we have

$$
\frac{1}{N} \sum_{k=1}^{N} b_{k} \phi\left(b_{k}\right) \geq \frac{1}{N} \sum_{n \in M \cap\left[e^{2 C}, N\right]} b_{n} \phi\left(b_{n}\right) \geq \frac{\phi(C)}{N} \sum_{n \in M \cap\left[e^{2 C}, N\right]} b_{n}=\frac{\phi(C)}{N} \sum_{n \in\left[e^{2 C}, N\right]} b_{n} .
$$


This together with (4.1) and (4.8) implies

$$
\begin{aligned}
\liminf _{N \rightarrow \infty} \frac{1}{N} \sum_{k=1}^{N} b_{n} \phi\left(b_{n}\right) & \geq \phi(C)\left(\lim _{N \rightarrow \infty} \frac{1}{N} \sum_{k=1}^{N} \Lambda^{\prime}(n)-\limsup _{N \rightarrow \infty} \frac{1}{N} \sum_{k=1}^{N}\left|\Lambda^{\prime}(n)-b_{n}\right|\right) \\
& \geq(1-2 d) \phi(C) .
\end{aligned}
$$

Since $C>0$ was arbitrary, we obtain $\lim _{N \rightarrow \infty} \frac{1}{N} \sum_{k=1}^{N} b_{n} \phi\left(b_{n}\right)=\infty$ and hence $\left(b_{n}\right) \notin \mathscr{A}$.

\section{AVERAGES ALONG THE PRIMES FOR OPERATORS ON $L^{r}, 1<r<\infty$.}

We now want to extend the convergence of the averages along the primes, as in Theorem 4.1(ii), to positive contractions of $L^{r}, 1<r<\infty$, and to invertible operators $T$ on $L^{r}$ with $T$ and $T^{-1}$ power-bounded.

Definition 5.1. A linear operator on (the real or complex) $L^{r}(\Omega, \Sigma, \mu)$ of a $\sigma$-finite measure space is called a Lamperti operator (or disjointness preserving) if it preserves disjointness of supports, i.e., $T f$ and $T g$ have disjoint supports if $f$ and $g$ have disjoint supports.

Definition 5.2. $T$ on $L^{r}$ is called a quasi-isometry if there exist constants $c_{1}$ and $c_{2}$, and an increasing sequence of positive integers $\left(M_{n}\right)_{n \geq 1}$ such that for every $f \in L^{r}$ we have

$$
c_{1}\|f\|^{r} \leq \frac{1}{M_{n}} \sum_{k=1}^{M_{n}}\left\|T^{k} f\right\|^{r} \leq c_{2}\|f\|^{r}, \quad \forall n \geq 1 .
$$

If $T$ is invertible with both $T$ and $T^{-1}$ power-bounded, then $\|f\| \leq K\left\|T^{k} f\right\|$, so $T$ is a quasi-isometry. If $T$ is similar to a quasi-isometry, it is a quasi-isometry.

Proposition 5.1. Let $(\Omega, \Sigma, \mu)$ be a $\sigma$-finite measure space and fix $1<r<\infty$. Let $T$ be a Lamperti quasi-isometry on $L^{r}(\mu)$. Then there exists $C>0$ such that for every $f \in L^{r}(\mu)$

$$
\left\|\sup _{n}\left|\frac{1}{n} \sum_{k=1}^{n} T^{k} f\right|\right\|_{r} \leq C\|f\|_{r}
$$

and $\frac{1}{n} \sum_{k=1}^{n} T^{k} f$ converges a.e. and in norm.

Proof. The maximal inequality is obtained by transfering the maximal inequality for the shift on $\mathbb{Z}$, using [25, Theorem 2.1]. The a.e. convergence follows from [25] - the needed inequality (2.7) there was proved by Bourgain [8]. Together with (5.1), the a.e. convergence yields the norm convergence.

Theorem 5.1. Let $(\Omega, \Sigma, \mu)$ be a $\sigma$-finite measure space and fix $1<r<\infty$. Let $T$ be a linear operator on $L^{r}(\mu)$ satisfying one of the following conditions:

(i) $T$ is a positive contraction or a Lamperti contraction.

(ii) $T$ is an isometry.

(iii) $T$ is invertible, with both $T$ and $T^{-1}$ positive and power-bounded.

(iv) $T$ is a Lamperti quasi-isometry.

Then for every $f \in L^{r}$ we have convergence in norm of $\frac{1}{n} \sum_{j=1}^{n} T^{p_{j}} f$ and of $\frac{1}{N} \sum_{j=1}^{\pi(N)} \log p_{j} T^{p_{j}} f$.

Proof. We first show that it is enough to prove the theorem when $T$ satisfies (iv).

If $T$ satisfies (iii), then $T$ is a quasi-isometry as noted above, and the positivity yields that $T$ is Lamperti, by Kan [27, Proposition 3.1], so (iv) is satisfied. 
If $T$ satisfies (ii) and $r \neq 2$, then the isometry $T$ is a Lamperti operator [29, Theorem 3.1] ( $T$ need not be positive). When $r=2$ the theorem follows from Theorem 4.1 or Theorem 4.2.

When $T$ is a positive contraction, we use Akcoglu's dilation of $T$ to a positive isometry (see [1]), so the theorem in that case follows from its conclusion for isometries. When $T$ is a Lamperti contraction, it can be dilated to a (Lamperti) isometry by Kan's dilation [27, Theorem 4.4], so the theorem follows from its conclusion for isometries.

Jones, Olsen and Wierdl [25] proved that if $T$ is a Lamperti quasi-isometry on $L^{r}$, then $\frac{1}{n} \sum_{j=1}^{n} T^{p_{j}} f$ converges a.e. for every $f \in L^{r}$. However, in their proof they refer to [43] for the maximal inequality needed in their formula (2.8); but the maximal inequality proved in [43] is for the modulated averages, modulated by $\left(\Lambda^{\prime}(k)\right)$. (Their formula (2.7) is proved in [8] for the averages along the primes). We are grateful to Máté Wierdl for showing us the equivalence of these maximal inequalities (see details in the Appendix). With the correct maximal inequality, when transferred to $L^{r}$ using [25, Theorem 2.1], we have that $\sup _{n}\left|\frac{1}{n} \sum_{j=1}^{n} T^{p_{j}} f\right|$ is in $L^{r}$, so the a.e. convergence proved in [25] implies norm convergence, by Lebesgue's bounded convergence theorem. This proves the theorem for the averages along the primes. The other convergence follows from Proposition 4.1.

Remark 5.1. 1. Theorem 5.1 applies also to operators on $L^{r}$ which are similar to an operator satisfying one of the assumptions (i)-(iv).

2. Kan [27, Example 3.1] has an example (on $\ell^{r}(\mathbb{Z})$ ) of a positive invertible $T$ with $T$ and $T^{-1}$ powerbounded, such that $T$ and $T^{-1}$ are not Lamperti ( $T^{-1}$ is not positive). Assani [4] constructed for each $1<r<\infty$ an invertible doubly power-bounded operator $T$ on $L^{r}[0,1]$ which does not satisfy the pointwise ergodic theorem; hence, by [27, Theorem 5.3], $T$ is not Lamperti (and none of its powers is).

3. Gillespie [22, p. 251] constructed, for any locally compact $\sigma$-compact Abelian group $G$ and $r \neq 2$, an invertible $T$ on the complex $L^{r}(G)$ with $T$ and $T^{-1}$ power-bounded, which is not similar to an isometry (unlike the result for $r=2$ [40]), though $T^{3}$ is an isometry. We will show below that his construction yields $T$ which in fact is not similar to a Lamperti operator.

We recall the structure of Lamperti operators [27, Theorem 4.1 and Proposition 4.1]: Let $T$ be a Lamperti operator on $L^{r}(\Omega, \Sigma, \mu)$. Then there exist a non-singular $\sigma$-endomorphism $\Phi_{0}$ of $\Sigma$, which induces a positive operator $\Phi$ on measurable functions, and a finite function $h$, such that $T f(\omega)=$ $h(\omega) \cdot \Phi f(\omega)$. This representation yields

$$
T^{n} f=\left[h \cdot \Phi h \cdots \Phi^{n-1} h\right] \cdot \Phi^{n} f, \quad f \in L^{r}(\Omega, \mu), \quad n \in \mathbb{N} .
$$

For the analysis of the spectrum of invertible Lamperti operators we need the following extension of [22, Theorem 3.1], where $r \neq 2$ is assumed only for identifying isometries as Lamperti operators.

Theorem 5.2. Let $1<r<\infty$, and let $T$ be an invertible Lamperti operator on the complex $L^{r}$ with representation $T f=h \cdot \Phi f$, satisfying $\sup _{n \in \mathbb{Z}}\left\|T^{n}\right\|<\infty$. If $\Phi \neq I$, then there exists a set $\sigma \in \Sigma$ with $0<\mu(\sigma)<\infty$ such that $\sigma$ and $\Phi_{0}(\sigma)$ are disjoint. Hence the set

$$
\mathscr{M}:=\left\{1 \leq m \in \mathbb{N}: \exists \sigma_{m} \in \Sigma, 0<\mu\left(\sigma_{m}\right)<\infty, \quad \sigma_{m}, \Phi_{0}\left(\sigma_{m}\right), \ldots, \Phi_{0}^{m}\left(\sigma_{m}\right) \text { are disjoint }\right\}
$$

is not empty.

(a) If $\mathscr{M}=\mathbb{N}$, then $\sigma(T)=\mathbb{T}$. 
(b) If $\mathscr{M} \neq \mathbb{N}$, then $\mathscr{M}$ is finite, with $m_{0} \geq 1$ points, and $\sigma(T)$ contains $m_{0}+1$ distinct points equally spaced on $\mathbb{T}$.

Proof. The proof that $\mathscr{M}$ is not empty is the same as in [22, Theorem 3.1].

(a) We adapt the proof of [22]. If $\mathscr{M}=\mathbb{N}$, then for every $m \in \mathbb{N}$ there is $\sigma_{m} \in \Sigma$ with $1_{\sigma_{m}} \in L^{r}$, such that $\left.\left(\Phi_{0}^{k}\left(\sigma_{m}\right)\right)_{0 \leq k \leq m}\right)$ are disjoint. Hence, by (5.2), $\left(T^{k} 1_{\sigma_{m}}\right)_{0 \leq k \leq m}$ have disjoint supports.

Denote $C:=\sup _{n \in \mathbb{Z}}\left\|T^{n}\right\|$. Then for $f \in L^{r}$ we have

$$
\frac{1}{C}\|f\| \leq\left\|T^{k} f\right\| \leq C\|f\| .
$$

Fix $\lambda \in \mathbb{T}$, and put $f_{m}:=\sum_{n=0}^{m} \lambda^{-n} T^{n} 1_{\sigma_{m}}$. Disjointness of supports yields

$$
\left\|f_{m}\right\|^{r}=\sum_{n=0}^{m}\left\|T^{n} 1_{\sigma_{m}}\right\|^{r} \geq \frac{1}{C^{r}}(m+1)\left\|1_{\sigma_{m}}\right\|^{r}=\frac{m+1}{C^{r}} \mu\left(\sigma_{m}\right) .
$$

By definition $(\lambda I-T) f_{m}=\lambda 1_{\sigma_{m}}-\lambda^{-m} T^{m+1} 1_{\sigma_{m}}$, so

$$
\left\|(\lambda I-T) f_{m}\right\| \leq\left\|1_{\sigma_{m}}\right\|+\left\|T^{m+1} 1_{\sigma_{m}}\right\| \leq(C+1)\left\|1_{\sigma_{m}}\right\|=(C+1) \mu\left(\sigma_{m}\right)^{1 / r} .
$$

Thus

$$
\frac{\left\|(\lambda I-T) f_{m}\right\|}{\left\|f_{m}\right\|} \leq \frac{C(C+1)}{(m+1)^{1 / r}} \underset{m \rightarrow \infty}{\rightarrow} 0 .
$$

Hence $\lambda I-T$ cannot have a bounded inverse, and $\lambda \in \sigma(T)$.

(b) Obviously $n \in \mathscr{M}$ implies that $m<n$ is in $\mathscr{M}$, so if $\mathscr{M} \neq \mathbb{N}$ then $\mathscr{M}$ is finite, and $\mathscr{M}=$ $\left\{1,2, \ldots, m_{0}\right\}$. It is proved in [22, pp. 249-250] that there exists $\sigma \in \Sigma$ with $0<\mu(\sigma)<\infty$ such that $\sigma, \Phi_{0}(\sigma), \ldots, \Phi_{0}^{m_{0}}(\sigma)$ are disjoint and $\Phi_{0}^{m_{0}+1}(\sigma)=\sigma$.

Let $\Omega_{0}=\bigcup_{k=0}^{m_{0}} \Phi_{0}^{k}(\sigma)$. Since for $0 \leq k \leq m_{0}$, we have

$$
T 1_{\Phi_{0}^{k}(\sigma)}=h \cdot \Phi 1_{\Phi_{0}^{k}(\sigma)}=h \cdot 1_{\Phi_{0}^{k+1}(\sigma)} \quad\left(\text { addition } \bmod m_{0}\right),
$$

the subspace $X:=L^{r}\left(\Omega_{0}, \mu\right)$ is $T$-invariant, and we put $T_{0}:=T_{\mid X}$. Since $T$ is invertible, it follows form [27, Proposition 4.1] that $h \neq 0$ a.e., $\Phi_{0}(\Omega)=\Omega, \Phi_{0}$ and its induced operator $\Phi$ are invertible, $\Phi 1=1$, and $T^{-1}$ is given by

$$
T^{-1} f(\omega)=\frac{1}{\Phi^{-1} h(\omega)} \Phi^{-1} f(\omega) .
$$

By invertibility, $\left(\Phi_{0}^{-1}\right)^{k}(\sigma)=\Phi_{0}^{m_{0}+1-k}(\sigma)$, so we have that $X$ is invariant also under $T^{-1}$, and $T_{0}$ is invertible, with $T_{0}^{-1}=\left(T^{-1}\right)_{\mid X}$. Hence $T_{0}$ and $T_{0}^{-1}$ are power-bounded, so $\sigma\left(T_{0}\right) \subset \mathbb{T}$.

Let $\lambda=\mathrm{e}^{2 \pi i /\left(m_{0}+1\right)}$ be a primitive $\left(m_{0}+1\right)$ th root of unity, and define $S: X \longrightarrow X$, as in [22], by

$$
S f:=\sum_{k=0}^{m_{0}} \lambda^{-k} 1_{\Phi_{0}^{k}(\sigma)} f, \quad f \in X=L^{r}\left(\Omega_{0}\right) .
$$

It is computed in [22] (with $U$ there replaced by $T_{0}$ ) that $S^{-1} T_{0} S=\lambda T_{0}$. so $\sigma\left(T_{0}\right)=\lambda \sigma\left(T_{0}\right)$. Let $\gamma \in \sigma\left(T_{0}\right)$. Then $|\gamma|=1$, and the $m_{0}+1$ different points $\gamma, \lambda \gamma, \ldots, \lambda^{m_{0}} \gamma$ are in $\sigma\left(T_{0}\right)$. Since $\sigma\left(T_{0}\right) \subset \mathbb{T}$, all its points are in the approximate point spectrum of $T_{0}$ [41, p. 282]; hence the $m_{0}+1$ points $\gamma, \lambda \gamma, \ldots, \lambda^{m_{0}} \gamma$ are in $\sigma(T)$.

Lemma 5.1. Let $T$ and $S$ be similar bounded operators on a Banach space $X$. Then $\sigma(T)=\sigma(S)$ and $\sigma_{p}(T)=\sigma_{p}(S)$. 
Proof. This is a standard result. A direct computation from $T=V^{-1} S V$ yields $R(\lambda, T)=V^{-1} R(\lambda, S) V$ when $\lambda \in \rho(S)$, and $S(V f)=\lambda V f$ when $T f=\lambda f$. The lemma then follows.

Proposition 5.2. Let $\mu$ be the normalized Lebesgue measure on the unit circle $\mathbb{T}$, and fix $1<r<\infty, r \neq 2$. Then there exists an invertible operator on the complex $L^{r}(\mathbb{T}, \mu)$ such that both $T$ and $T^{-1}$ are powerbounded, but $T$ is not similar to a Lamperti operator (in particular, $T$ is not similar to an isometry), nor to a positive operator.

Proof. We will show that Gillespie's construction for $\mathbb{T}$ [22, p. 252] (repeated below for the sake of completeness) yields $T$ which is not similar to a Lamperti operator, nor to a positive one.

Let $\alpha \in(0,1)$ be irrational, and denote by $U_{\alpha}$ the rotation of $\mathbb{T}$ by the angle $2 \pi \alpha$. By [20] there exists a bounded operator $A_{\alpha}$ on $L^{r}(\mathbb{T}, \mu)$, with $\sigma\left(A_{\alpha}\right) \subset[0,2 \pi)$, such that $U_{\alpha}=\exp \left(i A_{\alpha}\right)$. Define $T:=\exp \left(i A_{\alpha} / 3\right)$, which is bounded on $L^{r}(\mathbb{T}, \mu)$. Then we have $\sigma(T) \subset\left\{\mathrm{e}^{i \theta}: \theta \in[0,2 \pi / 3]\right\}$. Putting $\alpha_{n}=n \alpha-[n \alpha]$, we obtain for $T$ the following representation as a multiplier:

$$
T\left(\sum_{n} a_{n} \mathrm{e}^{2 \pi i n t}\right):=\sum_{n} a_{n} \mathrm{e}^{2 \pi i \alpha_{n} / 3} \mathrm{e}^{2 \pi i n t} .
$$

Clearly $T^{3}=U_{\alpha}$. Hence $T$ is doubly power-bounded, with

$$
\sup _{n \in \mathbb{Z}}\left\|T^{n}\right\|=\max \left\{1,\|T\|,\left\|T^{2}\right\|\right\} .
$$

We will use Lemma 5.1 throughout the proof.

Assume that $T$ is similar to a Lamperti operator $S f=h \cdot \Phi f$. If $\Phi \neq I$, then $\sigma(S)=\sigma(T) \subset\left\{\mathrm{e}^{i \theta}\right.$ : $\theta \in[0,2 \pi / 3]\} \neq \mathbb{T}$ yields a contradiction to Theorem 5.2(b) (as in [22]). Thus $\Phi=I$, so $S f=h \cdot f$. By the similarity, $S$ is invertible with $S^{-1}$ power-bounded, so $|h| \equiv 1$ a.e and the multiplication operator $S$ is an invertible isometry. We now use an argument implicit in [22]. Since $S$ is a multiplication operator, so is $S^{3}$, and therefore $S^{3}$ is a spectral operator of scalar type, by [22, Theorem 3.1(i)]. Hence $U_{\alpha}$ is similar to a scalar-type spectral operator, say $U_{\alpha}=V^{-1} S^{3} V$. If $E(\cdot)$ is the spectral measure of $S^{3}$, then $F(\cdot):=V^{-1} E(\cdot) V$ is a spectral measure, and

$$
U_{\alpha}=V^{-1} S^{3} V=V^{-1}\left(\int_{\mathbb{T}} \lambda E(d \lambda)\right) V=\int_{\mathbb{T}} \lambda F(d \lambda),
$$

so $U_{\alpha}$ is also a spectral operator; but this contradicts Gillespie's [21, Theorem 2(ii)]. Hence $T$ is not similar to a Lamperti operator.

Assume now that $T$ is similar to a positive operator $S$. Then $S$ is power-bounded, and therefore satisfies Lotz's growth condition (G). Hence, by Lotz [31] (see [39, Theorem V.4.9, p. 327]) $\sigma(S)=\sigma(S) \cap \mathbb{T}$ is cyclic, i.e. for $\lambda \in \sigma(S)$, all its powers $\lambda^{k}$ are also in $\sigma(S)=\sigma(T)$. But since $\sigma(T)$ is a proper closed arc of $\mathbb{T}$, it cannot be cyclic. A different proof is by noting that since $L^{r}$ is reflexive, by Glück [23, Theorem 5.5] the point spectrum $\sigma_{p}(S)$ is cyclic. But the third powers of the eigenvalues of $T$ are $\left\{\mathrm{e}^{2 \pi i n \alpha}\right\}$, a dense subset of $\mathbb{T}$, so $\sigma_{p}(T)=\sigma_{p}(S)$, being a subset of a proper arc $\sigma(T)$, cannot be cyclic.

Remark 5.2. 1. Cuny gave a simple proof that Gillespie's $T$ is not similar to a multiplication operator, without using spectral operators; see Appendix B.

2. Since the cube of the above $T$ is a rotation, $T$ satisfies the pointwise ergodic theorem without being (similar to) a positive or a Lamperti operator (but $T^{3}$ is a positive Lamperti contraction).

3. Lemma 5.1 applies also when $S$ is on a different Banach space $Y$, and the similarity is by $V$ invertible from $X$ onto $Y$. Thus the proof shows that $T$ defined in Proposition 5.2 is not similar to a Lamperti or 
a positive operator on any other $L^{r}\left(\Omega^{\prime}, \mu^{\prime}\right)$ space. Note that $L^{r}(\Omega, \mu)$ is not isomorphic to $L^{q}\left(\Omega^{\prime}, \mu^{\prime}\right)$ if $r \neq q(1<r, q<\infty)$, since either their type or cotype constants, which are invariant under isomorphisms, are different [2, p. 154, Theorem 6.2.14] (see also [45, p. 98]).

4. Theorem 5.1 does not apply to $T$ of Proposition 5.2, because $T$ is not similar to any of the operators in Theorem 5.1.

We now show that positivity in (iii) of Theorem 5.1 (which implies the Lamperti property) is not necessary, so Theorem 5.3 below applies to $T$ of Proposition 5.2. The idea of the proof was suggested by Christophe Cuny.

Theorem 5.3. Let $(\Omega, \Sigma, \mu)$ be a $\sigma$-finite measure space and fix $1<r<\infty$. Let $T$ be an invertible operator on (the real or complex) $L^{r}(\mu)$, such that both $T$ and $T^{-1}$ are power-bounded. Then for every $f \in L^{r}$ we have norm convergence of $\frac{1}{n} \sum_{j=1}^{n} T^{p_{j}} f$ and of $\frac{1}{N} \sum_{j=1}^{\pi(N)} \log p_{j} T^{p_{j}} f$.

Proof. For the case $r=2$, see Remark 1 to Theorem 4.1 .

By Proposition 4.1 it is enough to prove the convergence of $S_{n} f:=\frac{1}{n} \sum_{j=1}^{n} \Lambda(j) T^{j} f$ for every $f \in L^{r}$. We put $C:=\sup _{k \in \mathbb{Z}}\left\|T^{k}\right\|$.

In order to prove that $S_{n} f$ is Cauchy, it is enough to prove that there is a constant $c(f)$ such that for every strictly increasing sequence $\left(N_{k}\right)$ of positive integers,

$$
\sum_{k=1}^{\infty}\left\|S_{N_{k+1}} f-S_{N_{k}} f\right\|_{r}^{r} \leq c(f)
$$

Fix $\left(N_{k}\right)$. For $K, M \geq 1$, we have

$$
\begin{gathered}
\sum_{k=1}^{K}\left\|S_{N_{k+1}} f-S_{N_{k}} f\right\|_{r}^{r} \leq \frac{C^{r}}{M} \sum_{m=1}^{M} \sum_{k=1}^{K}\left\|T^{m}\left(S_{N_{k+1}} f-S_{N_{k}} f\right)\right\|_{r}^{r}= \\
\frac{C^{r}}{M} \sum_{k=1}^{K} \sum_{m=1}^{M} \int\left|\frac{1}{N_{k+1}} \sum_{j=1}^{N_{k+1}} \Lambda(j) T^{j+m} f-\frac{1}{N_{k}} \sum_{j=1}^{N_{k}} \Lambda(j) T^{j+m} f\right|^{r} d \mu= \\
\frac{C^{r}}{M} \int \sum_{k=1}^{K} \sum_{m=1}^{M}\left|\frac{1}{N_{k+1}} \sum_{j=1}^{N_{k+1}} \Lambda(j) T^{j+m} f-\frac{1}{N_{k}} \sum_{j=1}^{N_{k}} \Lambda(j) T^{j+m} f\right|^{r} d \mu .
\end{gathered}
$$

We denote the above integrand by

$$
I_{K, M}(\omega):=\sum_{k=1}^{K} \sum_{m=1}^{M}\left|\frac{1}{N_{k+1}} \sum_{j=1}^{N_{k+1}} \Lambda(j) T^{j+m} f(\omega)-\frac{1}{N_{k}} \sum_{j=1}^{N_{k}} \Lambda(j) T^{j+m} f(\omega)\right|^{r} .
$$

For $\omega \in \Omega$, we define $g:=g_{M, K, \omega}$ on $\mathbb{Z}$ by $g(m)=T^{m} f(\omega)$ when $1 \leq m \leq N_{K+1}+M$ and $g(m)=0$ when $m \leq 0$ or $m>N_{K+1}+M$. We denote by $\sigma$ the shift on $\mathbb{Z}(\sigma(n)=n+1)$. Then

$$
g\left(\sigma^{j} m\right)=g(m+j)=T^{m+j} f(\omega) \text { for } 1 \leq m+j \leq N_{k+1}+M
$$

and $g\left(\sigma^{j} m\right)=0$ otherwise.

We first prove the theorem when $r>2$. We estimate the integrand $I_{K, M}(\omega)$ by

$$
\begin{gathered}
I_{K, M}(\omega)=\sum_{k=1}^{K} \sum_{m=1}^{M}\left|\frac{1}{N_{k+1}} \sum_{j=1}^{N_{k+1}} \Lambda(j) g\left(\sigma^{j} m\right)-\frac{1}{N_{k}} \sum_{j=1}^{N_{k}} \Lambda(j) g\left(\sigma^{j} m\right)\right|^{r} \leq \\
\sum_{k=1}^{K}\left\|\frac{1}{N_{k+1}} \sum_{j=1}^{N_{k+1}} \Lambda(j) g \circ \sigma^{j}-\frac{1}{N_{k}} \sum_{j=1}^{N_{k}} \Lambda(j) g \circ \sigma^{j}\right\|_{\ell^{r}(\mathbb{Z})}^{r} \cdot
\end{gathered}
$$


By the variation estimate of Zorin-Kranich [46, Theorem 1.1] with $q=r>2$ (which holds for complex functions in $\ell^{r}(\mathbb{Z})$ ), there exists a constant $c_{r}$ such that the last term above is bounded by $c_{r}^{r}\|g\|_{\ell^{r}(\mathbb{Z})}^{r}=$ $c_{r}^{r} \sum_{m=1}^{N_{K+1}+M}\left|T^{m} f(\omega)\right|^{r}$. Hence

$$
\sum_{k=1}^{K}\left\|S_{N_{k+1}} f-S_{N_{k}} f\right\|_{r}^{r} \leq \frac{C^{r}}{M} \int c_{r}^{r} \sum_{m=1}^{N_{K+1}+M}\left|T^{m} f\right|^{r} d \mu \leq \frac{N_{K+1}+M}{M} C^{2 r} c_{r}^{r}\|f\|_{r}^{r} .
$$

For $K$ fixed we let $M \rightarrow \infty$ and obtain $\sum_{k=1}^{K}\left\|S_{N_{k+1}} f-S_{N_{k}} f\right\|_{r}^{r} \leq C^{2 r} c_{r}^{r}\|f\|_{r}^{r}$. This proves (5.4) and yields that $\left(S_{n} f\right)$ is Cauchy.

We now prove the theorem when $1<r<2$. For $q>2$, we apply Hölder's inequality with exponent $q / r$ to the summation on $k$, and obtain the estimate

$$
\begin{gathered}
I_{K, M}(\omega)=\sum_{m=1}^{M} \sum_{k=1}^{K}\left|\frac{1}{N_{k+1}} \sum_{j=1}^{N_{k+1}} \Lambda(j) g\left(\sigma^{j} m\right)-\frac{1}{N_{k}} \sum_{j=1}^{N_{k}} \Lambda(j) g\left(\sigma^{j} m\right)\right|^{r} \leq \\
\sum_{m=1}^{M}\left(\sum_{k=1}^{K}\left|\frac{1}{N_{k+1}} \sum_{j=1}^{N_{k+1}} \Lambda(j) g\left(\sigma^{j} m\right)-\frac{1}{N_{k}} \sum_{j=1}^{N_{k}} \Lambda(j) g\left(\sigma^{j} m\right)\right|^{q}\right)^{r / q} K^{(q-r) / q}= \\
K^{(q-r) / q} \sum_{m=1}^{M}\left[\left(\sum_{k=1}^{K}\left|\frac{1}{N_{k+1}} \sum_{j=1}^{N_{k+1}} \Lambda(j) g\left(\sigma^{j} m\right)-\frac{1}{N_{k}} \sum_{j=1}^{N_{k}} \Lambda(j) g\left(\sigma^{j} m\right)\right|^{q}\right)^{1 / q}\right]^{r} .
\end{gathered}
$$

The expression in the square brackets is bounded by the $q$-variation norm $\mathscr{V}_{q}$ of the sequence $\left(\frac{1}{N} \sum_{j=1}^{N} \Lambda(j) g\left(\sigma^{j}(m)\right)_{N>0}\right.$. Hence, using [46, Theorem 1.1], we obtain that

$$
\begin{gathered}
I_{K, M}(\omega) \leq K^{(q-r) / q}\|\|\left(\frac{1}{N} \sum_{j=1}^{N} \Lambda(j) g\left(\sigma^{j}(m)\right)_{N}\left\|_{\mathscr{V}_{q}}\right\|_{\ell^{r}(\mathbb{Z})}^{r} \leq\right. \\
K^{(q-r) / q} c_{r, q}^{r}\|g\|_{\ell^{r}(\mathbb{Z})}^{r}=K^{(q-r) / q} c_{r, q}^{r} \sum_{m=1}^{N_{K+1}+M}\left|T^{m} f(\omega)\right|^{r} .
\end{gathered}
$$

Hence

$$
\sum_{k=1}^{K}\left\|S_{N_{k+1}} f-S_{N_{k}} f\right\|_{r}^{r} \leq \frac{C^{r}}{M} \int I_{K, M}(\omega) d \mu \leq \frac{C^{r}}{M} c_{r, q}^{r} K^{(q-r) / q} C^{r}\left(N_{K+1}+M\right)\|f\|_{r}^{r} .
$$

For fixed $K$ we let $M \rightarrow \infty$ and obtain

$$
\sum_{k=1}^{K}\left\|S_{N_{k+1}} f-S_{N_{k}} f\right\|_{r}^{r} \leq C^{2 r} c_{r, q}^{r}\|f\|_{r}^{r} K^{(q-r) / q}
$$

We did not obtain (5.4), but by the next lemma $\left(S_{N} f\right)_{N}$ is Cauchy.

Lemma 5.2. Let $1 \leq r<\infty$ and let $\left(f_{n}\right)_{n \geq 1}$ be a sequence in a normed space $X$. If there exist $L>0$ and $\delta \in(0,1)$ such that for every increasing subsequence $\left(n_{j}\right)$ and every $K \in \mathbb{N}$ we have

$$
\sum_{j=1}^{K}\left\|f_{n_{j+1}}-f_{n_{j}}\right\|^{r} \leq L \cdot K^{\delta}
$$

then $\left(f_{n}\right)$ is Cauchy in $X$.

Proof. If $\left(f_{n}\right)$ is not Cauchy, then for some $\varepsilon>0$ we can construct an increasing sequence $\left(n_{j}\right)$ with $\left\|f_{n_{2 j}}-f_{n_{2 j-1}}\right\|>\varepsilon$. Then for any $K$ we obtain $K \varepsilon^{r} \leq L(2 K)^{\delta}$, which yields a contradiction. 
Remark 5.3. 1. Lemma 5.2 and its use for proving Theorem 5.3 when $r<2$ are inspired by the approach of Bourgain in [8, p. 209] (see also [8, p. 220]). Bourgain's estimates were used in [25].

2. Guy Cohen noted that the proof of Theorem 5.3 is valid for non-invertible power-bounded $T$ if for some $C^{\prime}$ we have $\|f\|_{r} \leq C^{\prime}\left\|T^{m} f\right\|_{r}$ for every $f \in L^{r}$ (e.g. $T$ is any isometry), since invertibility is used only in the estimate $\left\|S_{N_{k+1}} f-S_{N_{k}} f\right\|_{r} \leq C\left\|T^{m}\left(S_{N_{k+1}} f-S_{N_{k}} f\right)\right\|_{r}$. In fact, the proof is valid when $T$ is a power-bounded quasi-isometry, provided we take $M$ from the sequence $\left(M_{n}\right)$ in the definition of quasi-isometry. It follows that the Lamperti condition of Theorem 5.1(iv) is not necessary when $T$ there is power-bounded.

3. Proposition 5.2 provides examples to which Theorem 5.3 applies while Theorem 5.1 does not.

4. Christophe Cuny has informed us that extending ideas of Assani [4], [5], it is possible to construct an invertible operator $T$ on $L^{r}[0,1]$ with $T$ and $T^{-1}$ power-bounded, such that for some function $f \in L^{r}$ the averages along the primes $\frac{1}{n} \sum_{j=1}^{n} T^{p_{j}} f$ are almost everywhere non-convergent. See [10, Appendix].

\section{Problems}

In this section we list some problems which arise from our results.

1. Is there a necessary and sufficient condition on a sequence $\left(a_{n}\right)_{n \in \mathbb{N}}$ which ensures the convergence $\left\|\frac{1}{n} \sum_{k=1}^{n} a_{k} T^{k} x\right\| \rightarrow 0$ for every weakly almost periodic $T$ and every flight vector of $T$ ? In particular, is $\left(a_{n}\right) \in \overline{\mathscr{A}}^{W_{1}}$ necessary? The sequences $\left.\Lambda(n)\right)$ and $\left.\Lambda^{\prime}(n)\right)$ are not in $\overline{\mathscr{A}}^{W_{1}}$, but we do not know whether they modulate all flight vectors of weakly almost periodic operators; they modulate all almost periodic operators, by [30, Proposition 1.4].

2. Is Proposition 3.1 true for power-bounded operators in $H$ ? The answer is probably "NO", since the Blum-Hanson theorem fails, by Müller-Tomilov [34].

3. If $\sup _{n} \frac{1}{n} \sum_{k=1}^{n}\left|a_{k}\right|<\infty$ and $c(1):=\lim \frac{1}{n} \sum_{k=1}^{n} a_{k}$ exists, does a modulate all weakly stable operators in $H$ (extending Corollary 3.1)? It might be true, with negative answer to the previous problem.

4. Is Theorem 4.1(ii) true also for power-bounded operators in H? In view of the recent positive result of ter Elst and Müller [18], proving convergence of the averages along the squares, the cubes and other polynomials, the answer might be "YES".

5. Is Theorem 5.1 true also for power-bounded positive Lamperti operators? The proof of Theorem 5.1 uses the pointwise convergence of averages along the primes, proved in [25] for operators satisfying (iv) of the theorem. For power-bounded positive Lamperti operators, without invertibility, we are looking for the norm convergence only.

\section{APPENDIX A: ON THE MAXIMAL INEQUALITY ALONG THE PRIMES}

\section{by Máté Wierdl}

The proof of the pointwise convergence of averages along the primes in [43] is based on transforming the problem to the equivalent one of convergence of modulated averages with the modified von Mangoldt weights $\left(\Lambda^{\prime}(k)\right)$. The next lemma is the abstraction of this equivalence.

Lemma 7.1. Let $\left(t_{k}\right)_{k>0}$ be a sequence of reals. Then $\frac{1}{n} \sum_{j=1}^{n} t_{p_{j}}$ converges if and only if $\frac{1}{N} \sum_{j=1}^{\pi(N)} t_{p_{j}} \log p_{j}$ converges.

Proof. Lemma 1 of [43] is valid when then sequence $f\left(T^{a} x\right)$ is replaced by a sequence $\left(t_{a}\right)$, with the same proof. Then use the remark following the statement of [43, Lemma 1]. 
In [43] a maximal inequality was proved for the $\Lambda^{\prime}$-modulated averages. We show below that this inequality yields (is equivalent to) the maximal inequality for the averages along the primes.

Lemma 7.2. There exists a constant $c_{1}$ such that for any sequence of reals $\left(t_{k}\right)_{k>0}$ we have

$$
\sup _{N} \frac{1}{N} \sum_{j=1}^{\pi(N)}\left|t_{p_{j}}\right| \log p_{j} \leq c_{1} \sup _{n} \frac{1}{n} \sum_{j=1}^{n}\left|t_{p_{j}}\right|
$$

Proof. Since $\frac{\pi(N) \log N}{N} \rightarrow 1$, we put $c_{1}:=\sup _{N} \frac{\pi(N) \log N}{N}<\infty$. Since $p_{\pi(N)} \leq N$, for $\left(t_{k}\right)$ non-negative we have

$$
\frac{1}{N} \sum_{j=1}^{\pi(N)} t_{p_{j}} \log p_{j} \leq \frac{1}{N} \sum_{j=1}^{\pi(N)} t_{p_{j}} \log N=\frac{\pi(N) \log N}{N} \frac{1}{\pi(N)} \sum_{j=1}^{\pi(N)} t_{p_{j}} \leq c_{1} \sup _{n} \frac{1}{n} \sum_{j=1}^{n} t_{p_{j}},
$$

which yields the desired inequality.

Lemma 7.3. There exist constants $c$ and $c_{2}$ such that for any sequence $\left(t_{k}\right)$ with $t^{*}:=\sup _{n} \frac{1}{n} \sum_{k=1}^{n}\left|t_{k}\right|<\infty$ we have

$$
\sup _{n} \frac{1}{n} \sum_{j=1}^{n}\left|t_{p_{j}}\right| \leq c_{2} t^{*}+c \sup _{N} \frac{1}{N} \sum_{j=1}^{\pi(N)}\left|t_{p_{j}}\right| \log p_{j}
$$

Proof. Define $c:=2 \sup _{N \geq 2} \frac{N}{\pi(N) \log N}$ and $c_{2}:=\sup \frac{\sqrt{N}}{\pi(N)}$. Denote $\mathbb{P}_{N}:=\{p \in \mathbb{P}, p \leq N\}$. We may assume $t_{k} \geq 0$. Then

$$
\frac{1}{\pi(N)} \sum_{p \in \mathbb{P}_{\sqrt{N}}} t_{p} \leq \frac{1}{\pi(N)} \sum_{k=1}^{[\sqrt{N}]} t_{k}=\frac{[\sqrt{N}]}{\pi(N)} \cdot \frac{1}{[\sqrt{N}]} \sum_{k=1}^{[\sqrt{N}]} t_{k} \leq c_{2} t^{*}
$$

and for $N>1$ we have

$$
\begin{gathered}
\frac{1}{\pi(N)} \sum_{\sqrt{N}<p \in \mathbb{P}_{N}} t_{p} \leq \frac{1}{\pi(N)} \sum_{\sqrt{N}<p \in \mathbb{P}_{N}} \frac{\log p}{\log \sqrt{N}} t_{p}= \\
\frac{N}{\pi(N) \frac{1}{2} \log N} \cdot \frac{1}{N} \sum_{p \in \mathbb{P}_{N}}(\log p) t_{p} \leq c \sup _{N} \frac{1}{N} \sum_{j=1}^{\pi(N)} t_{p_{j}} \log p_{j} .
\end{gathered}
$$

Putting the above together, we obtain (7.1).

Corollary 7.1. Let $(\Omega, \Sigma, \mu)$ be a $\sigma$-finite measure space and fix $1<r<\infty$. Let $T$ be a mean-bounded (e.g. power-bounded) operator on $L^{r}(\mu)$. If

$$
\left\|\sup _{n}\left|\frac{1}{n} \sum_{j=1}^{n} T^{p_{j}} f\right|\right\|_{L^{r}} \leq C_{1}\|f\|_{L^{r}}, \quad \forall f \in L^{r}
$$

then

$$
\left\|\sup _{n}\left|\frac{1}{N} \sum_{j=1}^{\pi(N)} \log p_{j} T^{p_{j}} f\right|\right\|_{L^{r}} \leq C_{2}\|f\|_{L^{r}}, \quad \forall f \in L^{r} .
$$

Conversely, if $T$ satisfies (7.3) and

$$
\left\|\sup _{n}\left|\frac{1}{n} \sum_{k=1}^{n} T^{k} f\right|\right\|_{L^{r}} \leq C_{0}\|f\|_{L^{r}} \quad \forall f \in L^{r},
$$

then (7.2) holds. 
The above corollary applies to $T$ induced by a measure preserving transformation, in particular to $T$ induced on $\ell^{r}(\mathbb{Z})$ or $\ell^{r}\left(\mathbb{Z}^{+}\right)$by the shift. It applies also to Lamperti quasi-isometries on $L^{r}$ (see Proposition 5.1).

Recently, Pavel Zorin-Kranich [46] proved an estimate for the $q$-variation of the modulated averages of the shift in $\ell^{r}(\mathbb{Z})$ along the primes, with modulation by the von Mangoldt function $\Lambda(k)$. Similar estimates when the modulation is by $\Lambda^{\prime}(k)$ are given by Mirek, Trojan and Zorin-Kranich [33]. These inequalities are equivalent to an inequality for the $q$-variation of the averages along the primes without modulation. The proof will be published elsewhere.

\section{APPENDIX B: ON CERTAIN MULTIPLIER OPERATORS IN $L^{p}, p \neq 2$}

\section{by Christophe Cuny}

Let $\mu$ be Lebesgue's measure on $[0,1)$, and identify $\mathbb{T}$ with $[0,1)$ by $t \rightarrow \mathrm{e}^{2 \pi i t}$. For $n \in \mathbb{Z}$, set $e_{n}(t)=$ $\mathrm{e}^{2 i \pi n t}, t \in[0,1)$.

Fix $2 \neq p \in(1, \infty)$ and let $\left(a_{n}\right)_{n \in \mathbb{Z}}$ be a sequence of unimodular complex numbers which is a multiplier in $L^{p}$, i.e. the operator

$$
T\left(\sum_{n \in \mathbb{Z}} c_{n}(f) e_{n}\right)=\sum_{n \in \mathbb{Z}} a_{n} c_{n}(f) e_{n}
$$

defines an invertible doubly power-bounded operator on $L^{p}$.

Proposition 8.1. If $2 \neq p \in(1, \infty)$ and the $a_{n}$ are all different, then the multiplier operator $T$ defined on $L^{p}$ by (8.1) is not similar to a multiplication operator $S f=h \cdot f$.

Proof. By contradiction; assume that there exist operators $S$ and $V$ such that $V$ is invertible, $S f=h \cdot f$ for some $h$, and $T=V^{-1} S V$. For $n \in \mathbb{Z}$, set $f_{n}:=V e_{n}$ and put $A_{n}:=\operatorname{support}\left(f_{n}\right)$. Then

$$
h \cdot f_{n}=S f_{n}=V T e_{n}=a_{n} f_{n}, \quad n \in \mathbb{N} .
$$

Hence $h \equiv a_{n}$ on $A_{n}$ ( $\mu$-almost everywhere). Since $a_{n} \neq a_{m}$ for $m \neq n \in \mathbb{Z}$ by assumption, $\mu\left(A_{n} \cap A_{m}\right)=0$. The sets $\left(A_{n}\right)_{n \in \mathbb{Z}}$ are therefore disjoint (modulo $\mu$ ). It follows that

$$
\left\|f_{1}+\ldots+f_{n}\right\|_{p}^{p}=\sum_{i=1}^{n}\left\|f_{i}\right\|_{p}^{p}
$$

For $C=\max \left\{\|V\|^{p},\left\|V^{-1}\right\|^{p}\right\}$, we have

$$
\left\|e_{1}+\ldots+e_{n}\right\|_{p}^{p} / C \leq\left\|f_{1}+\ldots+f_{n}\right\|_{p}^{p} \leq C\left\|e_{1}+\ldots+e_{n}\right\|_{p}^{p}
$$

and (using $\left|e_{n}\right| \equiv 1$ )

$$
n / C=\sum_{i=1}^{n}\left\|e_{i}\right\|_{p}^{p} / C \leq \sum_{i=1}^{n}\left\|f_{i}\right\|_{p}^{p} \leq C \sum_{i=1}^{n}\left\|e_{i}\right\|_{p}^{p}=C n .
$$

We obtain from the above that $n / C^{2} \leq\left\|e_{1}+\ldots+e_{n}\right\|_{p}^{p} \leq C^{2} n$, which yields a contradiction, since orthonormality yields

$$
\begin{gathered}
\left\|e_{1}+\ldots+e_{n}\right\|_{p}^{p} \geq\left\|e_{1}+\ldots+e_{n}\right\|_{2}^{p}=n^{p / 2}, \quad \text { when } p>2, \\
\left\|e_{1}+\ldots+e_{n}\right\|_{p}^{p} \leq\left\|e_{1}+\ldots+e_{n}\right\|_{2}^{p}=n^{p / 2}, \quad \text { when } 1<p<2 .
\end{gathered}
$$




\section{Acknowledgments}

The authors are grateful to Guy Cohen, Christophe Cuny, Máté Wierdl and Manfred Wolff for several helpful discussions. The second author is grateful to the University of Leipzig, where part of the research was carried out, for its support and hospitality.

\section{REFERENCES}

[1] M.A. Akcoglu, L. Sucheston, Dilations of positive contraction in $L^{p}$ spaces, Canadian Math. Bull. 20 (1977), $285-292$.

[2] F. Albiac, N. Kalton, Topics in Banach Space Theory, second edition, Springer Graduate Texts in Mathematics 233, Springer, Switzerland, 2016.

[3] T. Apostol, Introduction to Analytic Number Theory, Undergraduate Texts in Mathematics, Springer, New York Berlin Heidelberg, 1976.

[4] I. Assani, On the punctual and local ergodic theorem for nonpositive power-bounded operators on $L_{\mathbf{C}}^{p}[0,1], 1<p<\infty$, Proc. Amer. Math. Soc. 96 (1986), 306-310.

[5] I. Assani, Sur les opérateurs à puissances bornées et le théorème ergodique ponctuel dans $L^{p}[0,1], 1<p<\infty$, Canadian J. Math. 38 (1986), 937-946.

[6] D. Berend, M. Lin, J. Rosenblatt, A. Tempelman, Modulated and subsequential ergodic theorems in Hilbert and Banach spaces, Ergod. Theory Dyn. Syst. 22 (2002), 1653-1665.

[7] V. Bergelson, A. Del Junco, M. Lemańczyk , J. Rosenblatt, Rigidity and non-recurrence along sequences, Ergod. Theory Dyn. Syst. 34 (2014), 1464-1502.

[8] J. Bourgain, An approach to pointwise ergodic theorems, Springer Lecture Notes in Math. 1317 (1988), $204-223$.

[9] J. Bourgain, Pointwise ergodic theorems for arithmetic sets, Publ. Math. IHÉS, 69 (1989), 5-45.

[10] G. Cohen, Doubly power-bounded operators on $L^{p}, 2 \neq p>1$, J. Math. Anal. Appl. to appear.

[11] D. Çömez, M. Lin, J. Olsen, Weighted ergodic theorems for mean ergodic $L_{1}$ contractions, Trans. Amer. Math. Soc. 350 (1998), 101-117.

[12] C. Cuny, T. Eisner, B. Farkas, Wiener's lemma along primes and other subsequences, preprint, available at https://arxiv.org/abs/1701.00101v4.

[13] C. Cuny, M. Weber, Ergodic theorems with arithmetical weights, Israel J. Math. 217 (2017), 139-180.

[14] H. Davenport, Multiplicative Number Theory, 2nd edition, Graduate Texts in Mathematics 74, Springer, Berlin Heidelberg, 1980.

[15] T. Eisner, B. Farkas, M. Haase , R. Nagel, Operator Theoretic Aspects of Ergodic Theory, Springer Cham, Heidelberg New York, 2015.

[16] T. Eisner, S. Grivaux, Hilbertian Jamison sequences and rigid dynamical systems, J. Funct. Anal. 261 (2011), $2013-2052$.

[17] H. El Abdalaoui, J. Kulaga-Przymus, M. Lemańczyk, T. de la Rue, The Chowla and the Sarnak conjectures from ergodic theory point of view, Discrete Contin. Dyn. Syst. 37 (2017), 2899-2944

[18] A.F.M. ter Elst, V. Müller, A van der Corput-type lemma for power bounded operators, Math. Z. 285 (2017), $143-158$.

[19] N. Frantzikinakis, B. Host, B. Kra, Multiple recurrence and convergence for sequences related to the prime numbers, J. Reine Angew. Math. 611 (2007), 131-144.

[20] T. Gillespie, Logarithms of $L^{p}$ translations, Indiana Univ. Math. J. 24 (1975), 1037-1045.

[21] T. Gillespie, A spectral theorem for $L^{p}$ translations, J. London Math. Soc. 11 (1975), 499-508.

[22] T. Gillespie, Power-bounded invertible operators and invertible isometries on $L^{p}$ spaces, in Operator semigroups meet complex analysis, harmonic analysis and mathematical physics, Operator Theory Adv. Appl. 250, pp. 241-252, Birkhäuser, Basel, 2015.

[23] J. Glück, On the peripheral spectrum of positive operators, Positivity 20 (2016), 307-336.

[24] L. Jones, M. Lin, Ergodic theorems of weak mixing type, Proc. Amer. Math. Soc. 57 (1976), 50-52.

[25] R. Jones, J. Olsen, M. Wierdl, Subsequence ergodic theorems for $L^{p}$ contractions, Trans. Amer. Math. Soc. 331 (1992), 837-850.

[26] J. P. Kahane, Sur les coefficients de Fourier-Bohr, Studia Math. 21 (1961), 103-106.

[27] C.H. Kan, Ergodic properties of Lamperti operators, Canadian J. Math. 30 (1978), 1206-1214. 
[28] U. Krengel, Ergodic Theorems, De Gruyter, Berlin, 1985.

[29] J. Lamperti, On the isometries of certain function spaces, Pacific J. Math. 8 (1958), 459-466.

[30] M. Lin, J. Olsen, A. Tempelman, On modulated ergodic theorems for Dunford-Schwartz operators, Illinois J. Math. 43 (1999), 542-567.

[31] H. Lotz, Über das Spektrum positiver Operatoren, Math. Z. 108 (1968), 15-32.

[32] A. Michal, R. Davis, M. Wyman, Polygenic functions in general analysis, Ann. Scuola Norm. Super. Pisa 9 (1940), 97-107.

[33] M. Mirek, B. Trojan, P. Zorin-Kranich, Variational estimates for averages and truncated singular integrals along the prime numbers, Trans. Amer. Math. Soc. 369 (2017), 5403-5423.

[34] V. Müller, Y. Tomilov, Quasisimilarity of power bounded operators and Blum-Hanson property, J. Funct. Anal. 246 (2007), 385-399.

[35] R. Nair, On polynomials in primes and J. Bourgain's circle method approach to ergodic theorems, Ergodic Th. Dyn. Syst. 11 (1991), 485-499.

[36] R. Nair, On polynomials in primes and J. Bourgain's circle method approach to ergodic theorems II, Studia Math. 105 (1993), 207-233.

[37] K. Prachar, Primzahlverteilung, Grundlehren Math. Wiss. vol. 91, Springer, Berlin Göttingen Heidelberg, 1957.

[38] G. Rhin, Sur la répartition modulo 1 des suites $f(p)$, Acta Arith. 23 (1973), 217-248.

[39] H.H. Schaefer, Banach Lattices and Positive Operators, Springer, Berlin Heidelberg New York, 1974.

[40] B.Sz-Nagy, On uniformly bounded linear transformations in Hilbert space, Acta Sci. Math. (Szeged) 11 (1947), $152-157$.

[41] A. E. Taylor, D. C. Lay, Introduction to Functional Analysis, second edition, Wiley and sons, New York, 1980.

[42] A. Walfisz, Zur additiven Zahlentheorie, II, Mathematische Zeitschrift 40 (1936), 592-607.

[43] M. Wierdl, Pointwise ergodic theorem along the prime numbers, Israel J. Math. 64 (1988), 315-336.

[44] M. Wierdl, Almost everywhere convergence and recurrence along subsequences in ergodic theory, Ph.D. Thesis, 186 p., Ohio State University, 1989.

[45] P. Wojtaszczyk, Banach Spaces for Analysts, Cambridge Studies in Advanced Mathematics 25, Cambridge University Press, New York, 1991.

[46] P. Zorin-Kranich, Variation estimates for averages along the primes and polynomials, J. Funct. Anal. 268 (2015), $210-238$. 